\title{
CD8 T cells compensate for impaired humoral immunity in COVID-19 patients with hematologic cancer
}

\section{Erin Bange}

University of Pennsylvania

Nicholas Han

University of Pennsylvania

https://orcid.org/0000-0003-1410-9931

E. Paul Wileyto

University of Pennsylvania

Justin Kim

University of Pennsylvania

https://orcid.org/0000-0003-0774-8137

Sigrid Gouma

University of Pennsylvania

https://orcid.org/0000-0002-7853-8340

James Robinson

University of Pennsylvania

Allison Greenplate

University of Pennsylvania

Florence Porterfield

University of Pennsylvania

Olutosin Owoyemi

University of Pennsylvania

Karan Naik

University of Pennsylvania

Cathy Zheng

University of Pennsylvania

https://orcid.org/0000-0002-0092-5463

Michael Galantino

University of Pennsylvania

Ariel Weisman

University of Pennsylvania https://orcid.org/0000-0002-7187-304X

Carolin Ittner

University of Pennsylvania

\section{Emily Kugler}

University of Pennsylvania

Amy Baxter 
UPenn https://orcid.org/0000-0002-1555-0713

\section{Madison Weirick}

University of Pennsylvania

\section{Christopher McAllister}

University of Pennsylvania

\section{Ngolela Esther Babady}

Memorial Sloan Kettering Cancer Center

\section{Anita Kumar}

Memorial Sloan Kettering

\section{Adam Widman}

Memorial Sloan Kettering Cancer Center

\section{Susan Dewolf}

Memorial Sloan Kettering

\section{Sawsan Boutemine}

Memorial Sloan Kettering

\section{Charlotte Roberts}

University of Pennsylvania

\section{Krista Budzik}

University of Pennsylvania

\section{Susan Tollett}

University of Pennsylvania

\section{Carla Wright}

University of Pennsylvania

\section{Tara Perloff}

University of Pennsylvania

\section{Lova Sun}

University of Pennsylvania

\section{Divij Mathew}

University of Pennsylvania https://orcid.org/0000-0002-8323-7358

\section{Josephine Giles}

University of Pennsylvania

\section{Derek Oldridge}

University of Pennsylvania

https://orcid.org/0000-0003-2177-5633

\section{Jennifer Wu}

University of Pennsylvania

\section{Cecile Alanio}

University of Pennsylvania https://orcid.org/0000-0003-2785-7445

\section{Sharon Adamski}

University of Pennsylvania 


\section{Laura Vella}

Children's Hospital of Philadelphia

\section{Samuel Kerr}

University of Pennsylvania

\section{Justine Cohen}

Massachusetts General Hospital

\section{Randall Oyer}

University of Pennsylvania https://orcid.org/0000-0001-7554-4166

\section{Ryan Massa}

University of Pennsylvania

\section{Ivan Maillard}

University of Pennsylvania

\section{Kara Maxwell}

University of Pennsylvania https://orcid.org/0000-0001-8192-4202

\section{Peter Maslak}

MSKCC

\section{Robert Vonderheide}

University of Pennsylvania

\section{Jedd D. Wolchok}

Memorial Sloan Kettering Cancer Center https://orcid.org/0000-0001-6718-2222

\section{Scott Hensley}

University of Pennsylvania https://orcid.org/0000-0002-2928-7506

\section{E. Wherry}

University of Pennsylvania https://orcid.org/0000-0003-0477-1956

\section{Nuala Meyer}

$$
\text { UPenn }
$$

\section{Angela DeMichele}

University of Pennsylvania Perelman School of Medicine https://orcid.org/0000-0003-1297-4251

\section{Oluwatosin Oniyide}

University of Pennsylvania

\section{Roseline Agyekum}

University of Pennsylvania

\section{Thomas Dunn}

University of Pennsylvania

\section{Tiffanie Jones}

University of Pennsylvania Perelman School of Medicine

\section{Heather Giannini}

University of Pennsylvania

\section{Alfred Garfall}


University of Pennsylvania

John Reilly

UPenn

\section{Santosha Vardhana}

Memorial Sloan Kettering

Ronac Mamtani

University of Pennsylvania

Alexander Huang ( $\nabla$ alexander.huang@pennmedicine.upenn.edu )

University of Pennsylvania https://orcid.org/0000-0002-0099-0492

\section{Article}

Keywords: COVID-19, hematologic cancer, CD8 T cells

Posted Date: February 2nd, 2021

DOl: https://doi.org/10.21203/rs.3.rs-162289/v1

License: (9) This work is licensed under a Creative Commons Attribution 4.0 International License. Read Full License

Version of Record: A version of this preprint was published at Nature Medicine on May 20th, 2021. See the published version at https://doi.org/10.1038/s41591-021-01386-7. 


\section{CD8 T cells compensate for impaired humoral immunity in COVID-19 patients with hematologic cancer}

Erin M. Bange ${ }^{\star 1,2}$, Nicholas A. Han¹,3, Paul Wileyto2,7, Justin Y. Kim ${ }^{1,3}$, Sigrid Gouma ${ }^{16}$, James

Robinson ${ }^{2}$, Allison R. Greenplate ${ }^{3,13}$, Florence Porterfield ${ }^{1}$, Olutosin Owoyemi ${ }^{1}$, Karan Naik ${ }^{1}$, Cathy

Zheng $^{2}$, Michael Galantino ${ }^{2}$, Ariel R. Weisman ${ }^{9}$, Caroline A.G. Ittner ${ }^{9}$, Emily M. Kugler ${ }^{1}$, Amy E. J Widman ${ }^{5}$, Susan DeWolf5,Sawsan R Boutemine ${ }^{5}$, Charlotte Roberts ${ }^{2}$, Krista R Budzik², Susan Tollett², Carla Wright'2, Tara Perloff2,11, Lova Sun,2, Divij Mathew ${ }^{3,13}$, Josephine R. Giles³,13,15, Derek A. Oldridge $^{3,14}$, Jennifer E. Wu $u^{3,13,15}$, Cécile Alanio ${ }^{3,13,15}$, Sharon Adamski3i,13, Alfred L. Garfall1,2, Laura Vella ${ }^{17}$, Samuel J. Kerr ${ }^{2,12}$, Justine V. Cohen ${ }^{2,11}$, Randall A. Oyer ${ }^{2,12}$, Ryan Massa ${ }^{1,2,10,}$, Ivan P. Maillard ${ }^{1,2}$, The UPenn COVID Processing Unit, Kara N. Maxwell ${ }^{1,2}$, John P. Reilly ${ }^{9}$, Peter G. Maslak ${ }^{5,6}$, Robert H. Vonderheide ${ }^{2,3,15}$, Jedd D. Wolchok ${ }^{4,5}$, Scott E. Hensley3,16, E. John Wherry ${ }^{3,13,15}$, Nuala Meyer ${ }^{3,9}$, Angela

M. DeMichele ${ }^{1,2}$, Santosha A. Vardhana ${ }^{ \pm * 4,5,15}$, Ronac Mamtani ${ }^{\star * 1,2}$, Alexander C. Huang ${ }^{ \pm \star 1,2,3,15}$

${ }^{1}$ Division of Hematology/Oncology, Department of Medicine, Perelman School of Medicine, University of Pennsylvania

${ }^{2}$ Abramson Cancer Center, University of Pennsylvania

${ }^{3}$ Institute for Immunology, Perelman School of Medicine, University of Pennsylvania

${ }^{4}$ Human Oncology and Pathogenesis Program, Memorial Sloan Kettering Cancer Center

${ }^{5}$ Department of Medicine, Memorial Sloan Kettering Cancer Center

${ }^{6}$ Department of Laboratory Medicine, Memorial Sloan Kettering Cancer Center

7 Department of Biostatistics, Epidemiology, and Informatics, Perelman School of Medicine, University of Pennsylvania

${ }^{8}$ Division of Hematology/Oncology, Department of Medicine, Perelman School of Medicine, Presbyterian Hospital

${ }^{9}$ Division of Pulmonary and Critical Care, Department of Medicine, Perelman School of Medicine, University of Pennsylvania

${ }^{10}$ Division of Hematology/Oncology, Department of Medicine, Perelman School of Medicine, Presbyterian Hospital

11 Division of Hematology/Oncology, Department of Medicine, Perelman School of Medicine, Pennsylvania Hospital

12 Division of Hematology/Oncology, Department of Medicine, Lancaster General Hospital

${ }^{13}$ Department of Systems Pharmacology and Translational Therapeutics, Perelman School of Medicine, University of Pennsylvania

${ }^{14}$ Department of Pathology and Laboratory Medicine, Perelman School of Medicine, University of Pennsylvania

${ }^{15}$ Parker Institute for Cancer Immunotherapy

${ }^{16}$ Department of Microbiology, Perelman School of Medicine, University of Pennsylvania

17 Department of Pediatrics, Perelman School of Medicine, Children's Hospital of Philadelphia

${ }^{*}$ These authors contributed equally to this work

${ }^{ \pm}$Co-Corresponding author(s):Address correspondence to S.V. (vardhans@mskcc.org), R.M. (ronac.mamtani@pennmedicine.upenn.edu), or A.C.H (alexander.huang@pennmedicine.upenn.edu) 
Cancer patients have increased morbidity and mortality from Coronavirus Disease 2019 (COVID-19), but the underlying immune mechanisms are unknown. In a cohort of 100 cancer patients hospitalized for COVID-19 at the University of Pennsylvania Health System, we found that patients with hematologic cancers had a significantly higher mortality relative to patients with solid cancers after accounting for confounders including ECOG performance status and active cancer status. We performed flow cytometric and serologic analyses of 106 cancer patients and 113 non-cancer controls from two additional cohorts at Penn and Memorial Sloan

55 Kettering Cancer Center. Patients with solid cancers exhibited an immune phenotype similar to non-cancer patients during acute COVID-19 whereas patients with hematologic cancers had

57 significant impairment of B cells and SARS-CoV-2-specific antibody responses. High dimensional analysis of flow cytometric data revealed 5 distinct immune phenotypes. An immune phenotype characterized by CD8 T cell depletion was associated with a high viral load and the highest mortality of $71 \%$, among all cancer patients. In contrast, despite impaired B cell responses, patients with hematologic cancers and preserved CD8 T cells had a lower viral load and mortality. These data highlight the importance of CD8 T cells in acute COVID-19, particularly in the setting of impaired humoral immunity. Further, depletion of B cells with antiCD20 therapy resulted in almost complete abrogation of SARS-CoV-2-specific IgG and IgM antibodies, but was not associated with increased mortality compared to other hematologic cancers, when adequate CD8 T cells were present. Finally, higher CD8 T cell counts were associated with improved overall survival in patients with hematologic cancers. Thus, CD8 T cells likely compensate for deficient humoral immunity and influence clinical recovery of COVID19. These observations have important implications for cancer and COVID-19-directed treatments, immunosuppressive therapies, and for understanding the role of $\mathrm{B}$ and $\mathrm{T}$ cells in 71 acute COVID-19. 


\section{Main Text}

73 Severe illness affects up to $20 \%$ of those hospitalized with Coronavirus Disease 2019 (COVID-

$7419)^{1}$ and is manifested by acute respiratory distress syndrome (ARDS), multi-organ failure,

75 and/or death ${ }^{2}$. Severe disease has been linked to immune dysregulation, including deficiency in

76 the production of type I and type III interferons ${ }^{3-5}$, marked lymphopenia ${ }^{6-10}$, and a paradoxical

77 increase in pro-inflammatory cytokines, such as TNFa, IL-1 $\beta$, and IL-6 $3,6,11-15$. In addition, alteration of the lymphocyte compartments has been reported during COVID-19 with increases in activated CD4 and CD8 T cells ${ }^{15-18}$, skewing of CD8 T cells towards effector ${ }^{16,17}$ and exhausted phenotypes ${ }^{18}$, and increased differentiation of CD4 T cells towards the Th17

81 lineage ${ }^{17,19}$. Despite these marked alterations in their T cell compartment, COVID-19 patients

82 have robust plasmablast responses ${ }^{15,20}$, and the majority of patients generate $\operatorname{lgM}$ and $\lg G$ antibodies to SARS-CoV-2 over the course of disease ${ }^{20-22}$. More recently, integrated and multiomic analyses have highlighted the tremendous heterogeneity of the human immune response to SARS-CoV-2, with distinct immunophenotypes that are associated with COVID-19 disease severity and disease trajectory ${ }^{5,11,12,15,16}$. Understanding how clinical features, particularly

87 patient comorbidity, impact host immune responses to SARS-CoV-2 will elucidate determinants of immunotype and disease severity.

Cancer patients have an increased risk of severe illness from COVID-1923-26 with an estimated case fatality rate of $25 \%{ }^{27}$ compared to $2.7 \%$ in the general population ${ }^{28}$. Importantly, cancer is a heterogeneous disease with even higher mortality rates reported for patients with

92 particular subtypes of cancer. For example, several cohort and registry studies have

93 demonstrated particularly poor outcomes among patients with hematologic cancers, with 94 mortality rates as high as $55 \%^{23,26,29-37}$. However, it remains unknown whether the increased mortality by cancer subtype is independent of the confounding effects of other prognostic factors such as Eastern Cooperative Oncology Group (ECOG) performance status, active cancer 
status, and cancer therapy. Further, data is limited on the immune landscape of cancer patients; whether components of cellular and humoral immunity are compromised, the impact of immunemodulating therapies such as B cell depleting therapy, and how these factors influence mortality in the setting of COVID-19 is also not known. To address these questions, we studied three cohorts of cancer patients with acute COVID-19 across two hospital systems to understand the immunologic determinants of COVID-19 mortality in cancer.

\section{Hematologic cancer is an independent risk factor of COVID-19 mortality}

To understand the clinical impact of COVID-19 on cancer patients, we first conducted a prospective multi-center observational cohort study of cancer patients hospitalized with COVID19 (COVID-19 Outcomes in Patients with Cancer, COPE). Between April 28 and September 15 2020, 114 patients with history of hematologic or solid tumor malignancy, and laboratoryconfirmed SARS-CoV-2 infection or presumed COVID-19 diagnosis, were enrolled across 4 hospitals in the University of Pennsylvania Health System. 14 patients were excluded from the analyses due to either low suspicion for COVID-19 infection, or benign tumor diagnosis. The median age of this cohort was 68 years, $52 \%$ were male, $54 \%$ Black, and $57 \%$ were current or former smokers (Table 1), reflecting the demographics of severe COVID-19 ${ }^{38,39}$. In terms of cancer-specific factors, $78 \%$ of patients had solid cancers, with prostate and breast cancers most prevalent; $46 \%$ had active cancer, defined as diagnosis or treatment within 6 months; and $49 \%$ had a recorded ECOG performance status of 2 or higher (Table 1). During follow up, $48 \%$ of subjects required ICU level care, and $38 \%$ of patients died within 30 days of admission (Table 2), consistent with previously reported rates for severe COVID-19 in this population ${ }^{30,34,}$ 37.

To understand key determinants of COVID-19 disease severity, we performed univariate analysis to identify factors associated with all-cause mortality within 30 days of discharge. We included relevant covariates, including patient factors such as age, race, gender, and smoking 
122 history (ever versus never) ${ }^{2}$, 38-40; cancer-specific factors including ECOG performance status ${ }^{35}$, 123 status of cancer (e.g., active versus remission) ${ }^{36,36}$; cancer type (e.g., heme versus solid 124 cancer) ${ }^{29}, 34,36,41,42$; and cancer treatment ${ }^{26,37}$. Increased mortality was significantly associated 125 with prior or current smoking ( $p=0.028)$, poor ECOG performance (ECOG 3-4, $p=0.001)$, and 126 active cancer status ( $p=0.024)$ (Fig. 1). In addition, patients with hematologic cancers (mostly

127 lymphoma and leukemia), appeared to have an increased risk of mortality relative to solid 128 cancers (54\% versus $33 \%$ respectively, $p=0.075)$ (Table 3 ). This is consistent with recent data

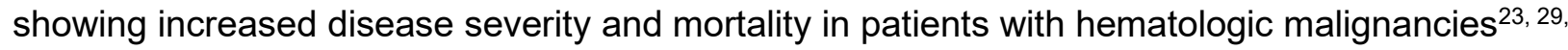
34-36, 41 . Notably, cancer treatment, including cytotoxic chemotherapy, was not significantly associated with COVID-19 mortality, also consistent with published literature in patients with cancer $^{29,} 30,34,36,41$.

To determine whether the increased mortality observed in patients with hematologic malignancy was independent of potential confounding effects from smoking history, poor ECOG performance, and active cancer, which were not corrected for in the prior studies, we performed multivariable logistic regression. Patients with hematologic cancers tended to be younger, male, less likely to have coexisting comorbidities, and more likely to have received recent cytotoxic chemotherapy (Supplemental Table 1). In this fully adjusted analysis, hematologic malignancy was strongly associated with mortality, in comparison to solid cancer (OR $3.3,95 \% \mathrm{Cl} 1.01$ 10.8, $p=0.048$ ) (Table 3). Similar results were observed in time-to-event analyses using Kaplan

141 Meyer methods (Fig. 2a, median overall survival (mOS) not reached for patients with solid cancers vs 47 days for patients with heme cancers, $p$-value $=0.030$ ) and Cox regression models

143 (Table 3, HR 2.56, 95\% Cl 1.19-5.54, $\mathrm{p}=0.017$ ). Moreover, patients with hematologic cancers 144 had higher levels of many inflammatory markers on admission laboratory testing, including 145 ferritin, IL-6, and LDH (Fig. 2b). There were no significant differences in CRP, fibrinogen, D146 dimer, lymphocyte counts, and neutrophil counts, while ESR was higher in patients with solid 
cancer (Extended Data Fig. 1 a,b). Thus, hematologic malignancy was an independent risk

148 factor of death, with signs of a dysregulated inflammatory response.

Hematologic cancer patients have an impaired SARS-CoV2-specific antibody response.

To understand the immune landscape in cancer patients, as compared to patients without cancer, we leveraged an observational study of hospitalized COVID-19 patients at the University of Pennsylvania Health System where blood was collected (MESSI-COVID) ${ }^{15}$. This analysis included 130 subjects with flow cytometric and/or serologic analysis. In particular, we

154 focused on 22 subjects with active cancer (Supplemental Tables 2, 3), including patients

155 undergoing cancer-directed therapies such as chemotherapy, immunotherapy, or B cell directed 156 therapies (Supplemental Table 4). Age, gender, and race were similarly distributed in COVID15719 patients with active cancer and those without, and both groups had a similar timeframe of 158 symptom onset and disease severity (Fig. 3a, Supplemental Table 2). However, cancer 159 patients had a higher all-cause mortality (36.4\% versus $11.1 \%$, Fig. 3a), consistent with our COPE clinical cohort, and what has been reported in other cohorts of COVID-19 patients ${ }^{23,26,29,}$ 16130.

As humoral immunity is critical for protective immunity against SARS-CoV-2, we hypothesized that a defect in SARS-CoV-2-specific antibodies may be associated with the 164 increase in mortality seen in patients with active cancer. We assessed the levels of $\operatorname{lgM}$ and IgG 165 antibodies that recognized the SARS-CoV-2 receptor binding domain (RBD), using an enzyme166 linked immunosorbent assay (ELISA) based approach ${ }^{43}, 44$. Cancer patients had significantly 167 decreased SARS-CoV-2-specific IgG and IgM responses compared to non-cancer patients 168 (Extended Data Fig. 2a). These differences were not due to the timing of SARS-CoV-2 169 infection as time from symptom onset was similar (Supplemental Table 2). As hematologic 170 malignancies directly involve the lymphoid and myeloid immune compartments, we suspected 171 that hematologic cancers may have an impaired humoral immunity against SARS-CoV-2. 
172 Indeed, the vast majority of hematologic cancer patients (6/7) had IgM and IgG levels below the

173 cutoff of positivity of 0.48 arbitrary units (Fig. 3b, Extended Data Fig. 2b). In contrast, those

174 with solid cancers had IgG and IgM antibody responses that were more comparable to patients

175 without cancer (Fig. 3b).

\section{A T cell-depleted immune phenotype is associated with COVID-19 mortality.}

177 Protective antibody responses require effective $T$ cell and B cell responses. We therefore 178 examined whether cancer patients had an altered cellular response to SARS-CoV-2. We first

179 performed exploratory high-dimensional analysis on the lymphocyte compartment of 45 patients 180 with COVID-19 including 37 non-cancer, 6 solid cancer, and 2 hematologic cancer patients.

181 UMAP (Uniform Manifold Approximation and Projection) representation of 27-parameter flow

182 cytometry data highlighted discrete islands of CD4 and CD8 T cells, and CD19+ B cells

183 (Extended Data Fig. 3a and Fig. 3c). To understand whether there were major global differences in lymphocytes between solid, hematologic, and non-cancer patients, we used the Earth Mover's Distance (EMD) metric $^{45}$ to calculate the distance between the UMAP projections for every pair of patients. Clustering on EMD values identified 5 clusters of patients with similar lymphocyte profiles (Fig. 3c). Differences between these clusters of patients were driven by both the distribution (Fig. 3d) and phenotype (Extended Data Fig. 3b and Fig. 3e) of CD4, CD8, and B cells. EMD cluster 1 was defined by depleted CD4 and B cells, increased CD8 T cells, and increased activation and effector markers, including PD-1, CX3CR1, Ki67, and HLADR (Extended Data Fig. 3b and Fig. 3 d,e). EMD cluster 3 had decreased T cell and B cells, 192 with an inactivated immune profile, and EMD Cluster 5 was depleted of both CD4 and CD8 T 193 cells, but had preserved B cells. In contrast, EMD cluster 4 was defined by robust

194 CCR7+CD27+ memory CD4 T cell responses and heterogenous B cell responses; EMD cluster 1952 had the most balanced responses, with CD4, CD8, and B cells represented (Fig. 3d,e and 196 Extended Data Fig. 3b). We then correlated these 5 patterns of immune responses with clinical 
and serological variables. EMD cluster 5 patients with depleted T cells had the highest mortality and disease severity, despite generating SARS-CoV-2-specific IgM and IgG antibodies (Fig. 3f, Extended Data Fig. 5d). In contrast, EMD clusters 2 and 4, with robust CD4 and/or CD8 T cell responses, had the lowest mortality and a low disease severity (Fig. 3f, Extended Data Fig. 5d). These findings suggest a key role for T-cell immunity in facilitating viral clearance, even in the presence of intact humoral immunity.

\section{Distinct immune landscape in hematologic cancer compared to solid cancer or no} cancer.

To further understand the immune response of patients with cancer and COVID-19, we explored the role of cancer subtype (solid tumor versus hematologic) on immune phenotype. Four out of the 6 solid cancer patients were in EMD cluster 2, with a balanced immune phenotype (Fig. 3e). In contrast, both hematologic cancer patients were in EMD cluster 1, which had marked depletion of CD4 and B cells. Indeed, UMAP projections showed that while solid cancer patients had an immune landscape similar to non-cancer patients, the two hematologic cancer patients demonstrated loss of islands associated with CD4 and B cells (Fig. $\mathbf{3 g}$ ). We then extended this analysis by measuring the frequency and phenotype of key lymphocyte populations in the entire MESSI-COVID cohort and healthy donor controls. COVID-19 patients with hematologic cancers had a significantly lower frequency of CD4 and B cells compared to solid cancer patients, noncancer patients, and healthy donors without COVID-19 (Fig. 3h). As T follicular helper cells (Tfh) and plasmablasts are critical in the generation of effective antibody responses, we assessed circulating Tfh and plasmablast responses. Although limited by sample size, patients with hematologic cancers had low circulating Tfh (PD1+ CXCR5+) and plasmablast responses $\left(C D 19+C D 27^{\text {hi }} C D 38^{\text {hi }}\right)$, and decreased CD138 expression (Extended Data Fig. 4a). Thus, patients with hematologic malignancy appear to have quantitative defects in CD4 and B cells that may be required for effective SARS-CoV-2-specific antibody responses. 
Patients with hematologic cancers had a preserved frequency of CD8 T cells. Therefore,

223 we wanted to determine whether there were qualitative changes within the CD8 T cell

224 compartment. We performed FlowSOM clustering analysis on non-naïve CD8 T cells from 118

225 COVID-19 patients and 30 healthy donors and visualized the clusters using UMAP. UMAP

226 clearly separated CX3CR1 and Tbet expressing effector cells from memory CD8 T cells

227 expressing CD27 and TCF-1 (Extended Data Fig. 4b and Fig. 3i). The effector island was

228 composed of CD45RA ${ }^{10} \mathrm{CD} 27^{\mathrm{lo}}$ effector memory cells (clusters 2 and 3 ) and CD45RA+ TEMRA

229 cells (cluster 1 ). The memory island was composed of CCR7 $7^{\text {lo }}$ transitional memory (cluster 5),

230 and effector memory cells (clusters 7 and 8), and CCR7 ${ }^{\text {hi }}$ central memory cells (cluster 9).

231 Activated cells, characterized by high HLA-DR, CD38, and Ki67 expression, were identified in

232 clusters 3, 4, and 5 (Extended Data Fig. 4c). Stem cell memory cells (cluster 10) and

233 exhausted phenotype CD8 T cells (cluster 6) were present, but at low frequencies of below

$0.5 \%$ (Data not shown).

We then compared the landscape of CD8 T cells in patients with and without cancer.

CD8 T cell subsets including central memory, effector memory, transitional memory and EMRA,

237 were similar between patients with and without cancer (Extended Data Fig. 4d). However,

238 UMAP representation of non-naïve CD8 data demonstrated preferential enrichment of cells

239 expressing HLA-DR and CD38 in cancer patients compared to non-cancer patients (Fig. 3j).

240 Indeed, cancer patients had higher frequencies of activated HLA-DR, CD38, and Ki67-

241 expressing FlowSOM clusters (clusters 3, 4, and 5) compared to non-cancer patients and

242 healthy donors (Extended Data Fig. 4e and Fig. 3k). When stratified by cancer type, the

243 increased HLA-DR and CD38 expression was restricted to the patients with hematologic

244 cancers; patients with solid cancers and those without cancer had comparable levels of

245 activation (Fig. 3I). Altogether, solid cancer patients with COVID-19 had an immune landscape

246 similar to non-cancer COVID-19 patients. In contrast, patients with hematologic malignancies

247 had defects in CD4, B cells, and humoral immunity but preserved and highly activated CD8 T 
cells, suggesting that CD8 T cells might at least partially compensate for blunted humoral immune responses in patients with hematologic malignancies.

250

251

\section{CD8 $T$ cell adequacy increases survival in the setting of impaired B cell and humoral} immunity in hematologic cancer.

Patients with hematologic cancer had significantly impaired humoral immunity and a mortality rate of 55\% (Table 2). We hypothesized that CD8 T cells partially compensated for defective humoral immunity and influenced survival in acute COVID-19. We tested this hypothesis in a cohort of cancer patients hospitalized with COVID-19 at the Memorial Sloan Kettering Cancer Center (MSKCC), which included a larger number of hematologic malignancies patients, including those treated with B cell depleting therapy. This cohort included 39 solid cancer patients and 45 hematologic cancer patients. The median age was 65 years, and in contrast to the MESSI cohort at Penn, $81 \%$ of the cohort was white (Fig. 4a, Supplementary Table 5,6). A significant portion of patients were treated with remdesevir and convalescent plasma $-21.4 \%$, and $46.4 \%$, respectively (Supplementary Table 5). Consistent with the Penn COPE and MESSI cohorts, patients with hematologic cancers did poorly, with a mortality rate of $44.4 \%$ (Fig. 4a, Supplementary Table 5). Clinical grade 12-parameter flow cytometry and serologic testing for SARS-CoV-2-specific antibodies were performed. In the MSKCC cohort, both CD4 and CD8 T cells were significantly decreased in patients with active solid and hematologic cancers, compared with patients in clinical remission (Extended Data Fig. 5a). Moreover, despite the fact that a substantial number of patients with hematologic cancers from the MSKCC cohort received convalescent plasma, they had a significant defect in SARS-CoV-2-specific IgG and IgM responses as compared to solid cancers (Extended Data Fig. 5b). This was independent of disease severity and viral load, as assessed by RT-PCR cycle threshold. (Extended Data Fig. 5c,d).

We performed high dimensional analyses on flow cytometry data that included 
273 information on CD4 T cells, CD8 T cells, and B cells. EMD and clustering of 20 solid cancer, 31

274 hematologic cancer, and 6 remission patients identified 4 immune phenotypes (Extended Data

275 Fig. 6a,b and Fig. 4b,c) that corresponded to the immune phenotypes 1,2,4, and 5 identified in

276 the Penn-MESSI cohort (Fig. 3c,d). The Penn phenotype 3, the only cluster that did not have

277 cancer patients, was not identified in the MSKCC cancer cohort. Consistent with the Penn data,

278 MSKCC EMD cluster 5, with depleted of CD4 and CD8 T cells and preserved B cells, had the

279 highest mortality of $71 \%$, and was associated with a high disease severity and viral load (Fig 280 4d).

Intriguingly, the clinical outcomes of patients with immune phenotype 4 was the greatest contributor to the overall mortality difference between patients with solid and liquid cancers; hematologic cancer patients with phenotype 4 had a mortality of $61 \%$ versus $9 \%$ in patients with solid cancers (Extended Data Fig. 7a), with a corresponding higher viral load as assessed by RT-PCR threshold cycle (Extended Data Fig. 7b). Immune phenotype 4 was characterized by robust CD4 responses and decreased, but still intact, CD8 responses (Extended Data Fig 6b). Within immune phenotype 4, patients with solid and hematologic cancers had similar CD4 and CD8 T cell counts (Extended Data Figure 7c). However, patients with hematologic cancers had near-complete abrogation of B cells (phenotype 4A), that corresponded with a mortality rate of 61\% (Extended Fig 7a and d). In contrast, patients with solid cancers had intact B cells counts 291 (phenotype 4B, Extended Data Fig 7a and d), with a mortality of 9\%. Thus, in a setting with 292 similar CD4 and CD8 T cell numbers, B cell depletion was associated with higher mortality; B 293 cells, therefore, likely play an important role in acute COVID-19.

Anti-CD20 therapy ( $\alpha C D 20)$ with rituximab or obinutuzumab-containing regimens depleted B cells with near-complete abrogation of SARS-CoV-2-specific IgG and IgM responses

296 (Fig. 4e). Notably, hematologic cancer patients on chemotherapy and solid cancer patients on immune checkpoint blockade also had significant depletion of B cells (Extended Data Fig. 8a). 
aCD20 therapy was not associated with quantitative changes in CD4 and CD8 T cells. However, patients treated with anti-CD20 therapy displayed dramatic reduction in CD4 and CD8 naïve and memory T cells, instead skewing towards effector differentiation and an activated HLADR+CD38+ phenotype (Extended Data Fig. 8b,c). Importantly, despite the loss of B cells and humoral immunity, aCD20 therapy was not associated with increased mortality, disease severity, or viral load when compared to chemotherapy or observation (Fig. 4f).

We sought to understand why aCD20 therapy was not associated with greater mortality in these patients. Patients treated with $\mathrm{aCD} 20$ therapy were restricted to immune phenotypes 1 and 4, characterized by depleted B cells (Fig $\mathbf{4 g}$ ). However, phenotype 1, characterized by preserved CD8 T cells, was associated with a lower mortality (Fig 4h). Indeed, aCD20 treated patients who survived their COVID-19 hospitalization had higher CD8 T cell counts (Fig 4i), and lower viral load (Extended Data Fig. 9a). We extended these analyses to other patients with hematologic cancers, including those on chemotherapy who also had quantitative (Extended Data Fig. 8a), and possibly qualitative B cell defects. Hematologic cancer patients who survived had higher CD8 T cell count (Fig. 4j), which was not seen in solid cancer patients (Extended Data Fig. 9b). Conversely, CD4 T cell counts were not associated with mortality, and higher B cell counts were associated with increased mortality (Extended Data Fig. 9b, Fig 4j). Thus, patients with hematologic cancers, in the setting of defective humoral immunity, were more highly dependent on adequate CD8 T cell counts than patients with solid cancers. Finally, Classification and Regression Tree Analysis (CART) identified a CD8 T cell level that was predictive of survival after COVID-19 in patients with hematologic cancers (Fig. 4k). Taken together, these findings suggest that CD8 T cells are critical for anti-viral immunity in hematologic malignancy patients and may at least partially mitigate the negative impact of B-cell depletion on COVID outcomes. 
324 A notable feature of the COVID-19 pandemic has been the dramatic heterogeneity in clinical

325 presentations and outcomes, yet mechanistic explanations for the wide variance in disease

326 severity have remained elusive. Early on, acute phase reactants and systemic cytokines were

327 implicated in patient outcomes ${ }^{46}$ and hospital stay and mortality were decreased by dexamethasone ${ }^{47}$, suggesting that an excessive host immune response might contribute to COVID-19 mortality. However, there were also indications that inadequate host immunity might contribute to adverse COVID-19 outcomes, including the association of lymphopenia with mortality as well as the potentially inferior outcomes of patients on chronic immunosuppression, such as patients with autoimmune diseases or organ transplant recipients ${ }^{48-51}$. Recent studies defined immune signatures associated with severe COVID-19, including activated CD4, CD8 T cells, plasmablasts, and robust antibody responses ${ }^{15,16,20,52}$. Nevertheless, the individual roles of these cell types in acute COVID-19 remained unclear. We speculated that investigating both the clinical outcomes and immunologic profile of cancer patients might shed valuable insight into how arms of the immune system contribute to viral control and mortality during COVID-19. Immune investigation in hematologic malignancies is especially relevant because the disease directly impacts the lymphoid and myeloid immune cells, and is commonly treated with myelosuppressive and B cell-depleting therapies including CD20 targeting antibodies.

343 after adjusting for ECOG performance and disease status. We observed a higher mortality rate 344 in patients with hematologic (53\%) versus solid cancers $(34 \%)$, which were substantially higher 345 than in the general population $(2.7 \%)^{28}$. The high mortality rates for hematologic cancer in this 346 study were consistent with a recent meta-analysis of 2,361 hospitalized patients with 347 hematologic cancer $(40 \%)^{53}$. This finding highlights the importance of transmission mitigation 
efforts for this vulnerable population ${ }^{54}$. Furthermore, we demonstrate that excess mortality observed with hematologic cancers persisted (HR 2.5) after adjustment for independent

350 predictors of cancer mortality, including age, smoking history, poor performance status, and 351 active or advanced disease. Adjustment for these factors was necessary to determine that the

352 increased mortality difference seen in hematologic cancer was in fact, driven by cancer subtype, 353 rather than differences in patient characteristics. These data can better inform hospitalized 354 patients with hematologic cancers of their expected outcomes, irrespective of performance 355 status or active cancer status, thereby improving decision-making between best supportive care 356 vaccinations to this very high-risk population.

Second, using high dimensional analyses, we define immune phenotypes associated with mortality during COVID-19. In particular, we identify the immune phenotype that drives the mortality difference between solid and liquid malignancy. A balanced immunity that included

362 CD4, CD8, and B cells responses (phenotypes 2 and 4b) was associated with low mortality. In contrast, an immune signature with robust $\mathrm{B}$ cell and humoral responses, but absent $\mathrm{T}$ cell responses (phenotype 5), was associated with the highest mortality (>60\%). A high mortality for patients with immune phenotype 5 was consistent in both the Penn and MSKCC cohorts, and in patients with solid cancer, hematologic cancer, and infected patients without cancer. Thus,

367 humoral immunity alone is often not sufficient in acute COVID-19. In fact, greater B cell responses was associated with higher mortality in both solid and liquid cancer. B cell responses may be a marker of disease severity, as seen with plasmablasts ${ }^{15,20}$ and neutrophils ${ }^{20,55,56}$ in severe COVID-19. Alternatively, some components of the B cell and humoral responses may be 371 aberrant and pathogenic, as may be the case with autoantibodies targeting type I interferons in 372 severe COVID-1957. Consistent with recent data ${ }^{58}$, patients with solid cancers had a similar cellular immune 
374 landscape and SARS-CoV-2-specific IgG responses as compared to patients without cancer.

375 Patients with hematologic cancers, however, had substantial defects in B cells and humoral

376 immunity. These defects were associated with a high mortality or $45 \%$, as compared to $25 \%$ in

377 solid cancers. This difference in survival was driven by immune phenotype 4, which was

378 characterized by robust CD4 T cell responses in conjunction with a diminished, but not absent

379 CD8 T response. This phenotype (phenotype 4B), in the setting of preserved B cells seen in

380 solid cancer patients, was associated with a low mortality of $9 \%$. However, this phenotype in the

381 setting of depleted B cells (phenotype 4A) seen in liquid cancer patients, was associated with a

382 mortality of $61 \%$. This highlights the fact that CD8 T cell responses that are normally sufficient

383 may no longer be adequate in the setting of compromised humoral immunity. Thus, CD4 or B

384 cells responses, in the absence of an intact CD8 T cell response, may not be sufficient to

control an acute SARS-CoV-2 infection. This is reminiscent of published data demonstrating

that uncoordinated immune responses in the elderly was associated with severe disease and

387 poor outcomes ${ }^{59}$.

Finally, by leveraging a population of COVID-19 patients in the setting of B cell depletion

389 (anti-CD20), we uncovered a critical protective role for CD8 T-cell responses. CD8 T cells are

390 known to be critical for viral clearance, particularly in response to higher viral inocula ${ }^{60}$. Recent

391 data from transgenic mouse models show that both CD4 and CD8 T cells are necessary for

392 optimal viral clearance of SARS-CoV-2 ${ }^{61}$. In patients treated with anti-CD20, absolute CD8 T

393 cell count, but not CD4 counts, was associated with survival from COVID-19 and lower viral

394 load. Although conclusions are limited by sample size, these data suggest that CD8 T cells play

395 a key role in limiting SARS-CoV-2, even in the absence of humoral immunity. Indeed, SARS-

396 CoV-2-specific CD8 T cell responses have been identified in acute and convalescent

397 individuals ${ }^{59,62-65}$. Further, in our cohort, absolute CD8 counts were predictive of outcomes in

398 the broader cohort of patients with hematologic malignancy. The compensatory role of CD8 T

399 cells was restricted to patients with hematologic, but not solid, malignancies. Thus, CD8 T cells 
likely play an important role in the setting of quantitative and qualitative B cell dysfunction in patients with lymphoma, multiple myeloma, and leukemia, undergoing anti-CD20, chemotherapy, or Bruton tyrosine kinase (BTK) inhibition. CD8 T cell counts may inform on the need for closer monitoring and a lower threshold for hospitalization in COVID-19 patients with hematologic malignancies. Furthermore, the clinical benefit of dexamethasone, which demonstrated an overall mortality benefit in hospitalized COVID-19+ patients but is known to suppress CD8 T cell responses ${ }^{66}$, should be investigated further in patients who recently received anti-CD20 therapy.

Recent analysis demonstrated that patients treated with B-cell depleting agents had the highest mortality rate, although this analysis did not account for whether the risk was modulated by CD8 count. Our findings do not exclude the possibility that B-cell depleting therapies may be associated with adverse outcomes in this population but rather extend these findings to suggest that an adequate CD8-dependent $T$ cell response is essential for patients in whom humoral immunity is compromised. We did, however, observe a profound depletion of both naive CD4 and CD8 T cells in patients receiving B-cell depleting agents. Naive T-cells, and particularly naive CD4 T cells, are known to require tonic TCR signaling driven by APC-presented selfantigens for persistence ${ }^{67,68}$. We speculate that depletion of functional B cells, particularly in the context of B cell depleting therapy, might lead to concomitant naive T cell depletion and a corresponding increase in the effector and activated CD8 T cells. Although the clinical relevance of naive T cell depletion in the setting of anti-CD20 is still unclear - it is notable that depletion of naive T cells in the elderly was associated with increased disease severity and poor outcomes $^{59}$.

Importantly, both B-cell depleting therapies and cytotoxic chemotherapy agents which can compromise the T-cell compartment are mainstays of lymphoma therapy. Both are administered, often in combination, with curative intent for patients with aggressive lymphomas, 
425 but also for debulking or palliation in patients with indolent lymphomas. Based on our data, we

426 would suggest that oncologists and patients considering treatment regimens that combine B cell

427 depletion with cytotoxic agents carefully weigh the associated increased risk of immune

428 dysregulation against the benefit of disease control when making an educated decision on

429 whether to initiate such treatments, particularly in non-curative settings.

$430 \quad$ Finally, our finding that CD8 T cell immunity is critical for survival in hematologic

431 malignancy patients with COVID-19 has profound implications for the vaccination of these

432 patients. Both the Pfizer and Moderna vaccines, as well as the Johnson and Johnson vaccine

433 currently under investigation, induce robust CD8 T cell responses in addition to humoral

434 responses ${ }^{69-71}$. Our findings suggest that vaccination of hematologic patients might enhance the

435 protective capacity of CD8 T-cells despite the likely absence of a humoral response. We are

436 conducting ongoing studies to monitor the immune profile of patients undergoing vaccination

437 prospectively to determine if this is the case. Ultimately, understanding how the immune

438 response relates to disease severity, cancer type, and cancer treatment will provide important

439 insight into the pathogenesis of and protective immunity from SARS-CoV-2, which may have

440 implications for the development and prioritization of therapeutics and vaccines in cancer

441 subpopulations.

442

443

444

445

446

447

448

449 
Methods:

451 COVID-19 Outcomes in Patients with Cancer, COPE

452 General Design/Patient Selection

We conducted a prospective cohort study of patients with cancer hospitalized with

454 COVID-19 (UPCC 06920). Informed consent was obtained from all patients. Adult patients with

455 a current or prior diagnosis of cancer and hospitalized with a probable or confirmed diagnosis of

456 COVID-19, as defined by the WHO criteria ${ }^{72}$, within the University of Pennsylvania Health

457 System (UPHS) between April 28, 2020 and September 15, 2020 were approached for consent.

458 Participating hospitals included the Hospital of the University of Pennsylvania, Presbyterian

459 Hospital, Pennsylvania Hospital, and Lancaster General Hospital. The index date was defined

460 as the first date of hospitalization within the health system for probable or confirmed COVID-19.

461 Repeat hospitalizations within 7 days of discharge were considered within the index admission.

462 Patients who died prior to being approached for consent were retrospectively enrolled. Patients

463 were followed from the index date to 30-days following their discharge or until death by any

464 cause. This study was approved by the institutional review boards of all participating sites.

465 Data Collection

466 Baseline characteristics including patient (age, gender, race/ethnicity, comorbidities, 467 smoking history, body mass index) and cancer (tumor type, most recent treatment, ECOG 468 performance status, active cancer status) factors as well as COVID-19 related clinical factors 469 including change in levels of care, complications, treatments such as need for mechanical 470 ventilation, laboratory values (complete blood counts with differentials and inflammatory 471 markers including LDH, CRP, ferritin, and IL-6), and final disposition were extracted by trained 472 research personnel using standardized abstraction protocols. Active cancer status was defined 473 by diagnosis or treatment within 6 months of admission date. Cancer treatment status was 474 determined by the most recent treatment within 3 months prior to admission date. 
The primary study endpoint was all-cause mortality within 30-days of hospital discharge.

476 Disease severity was categorized using the NIH ordinal scale including all post-hospitalization

477 categories: 1, hospitalized, not requiring supplemental oxygen but requiring ongoing medical

478 care; 2, hospitalized requiring any supplemental oxygen; 3, hospitalized requiring noninvasive

479 mechanical ventilation or use of high-flow oxygen devices; 4 , hospitalized receiving invasive

480 mechanical ventilation or extracorporeal membrane oxygenation (ECMO); 5 , death ${ }^{73}$, and was

481 assessed every 7 days throughout a patients admission.

482 Statistical Analysis

Cohort characteristics were compared using standard descriptive statistics. One-time

484 imputation of missing values for ECOG was done using the predicted mean value from an

ordinal logistic model (proportional odds) of complete data. The ordinal model was fitted with forward stepwise selection, with entry at $p=0.1$ and removal at 0.2 , using clinical variables expected to be correlated with ECOG performance status. Those variables included several items in the Charlson and severity score, and other clinical variables.

Univariate analyses examined demographic and clinical variables and cancer subtype admission. Odds ratios and $95 \% \mathrm{Cls}$ were used to generate the forest plot illustration. Baseline

492 laboratory tests were compared by cancer type using Mann Whitney tests and available RT493 PCR data was used to determine length of RT-PCR positivity by cancer type.

Rates of ICU admission and death were calculated for the overall cohort and 495 stratified by cancer subtype. A multivariate logistic model was used to examine the 496 adjusted effect of solid versus hematologic designation. Covariates included 497 demographic variables of age and sex (race was omitted for missing data). Covariates 498 also included clinical variables that attained a p-value of 0.1 in the univariate analyses. 499 The final model included age, sex, smoking status, active disease status, and ECOG 
performance status. A cox proportional hazards regression model was also performed to determine the association between cancer type and mortality and identically adjusted for age, sex, smoking status, active cancer status, and ECOG performance status. Overall survival (OS) was measured from date of hospitalization to last follow up or death and the median OS was estimated using Kaplan-Meier method and differences by cancer subtype compared using log-rank test.

\section{Immune profiling of patients hospitalized for COVID-19, MESSI}

Information on clinical cohort, sample processing, and flow cytometry is described in Mathew et al, Science 2020. Briefly, Patients admitted to the Hospital of the University of Pennsylvania with a positive SARS-CoV-2 PCR test were screened and approached for informed consent within 3 days of hospitalization. Peripheral blood was collected from all subjects and clinical data were abstracted from the electronic medical record into standardized case report forms. All participants or their surrogates provided informed consent in accordance with protocols approved by the regional ethical research boards and the Declaration of Helsinki. Methods for PBMC processing, flow cytometry, and antibodies used were previously described ${ }^{15}$.

\section{Serologic enzyme-linked immunosorbent assay (ELISA)}

ELISAs were completed using plates coated with the receptor binding domain (RBD) of the SARS-CoV-2 spike protein as previously described ${ }^{44}$. Briefly, Prior to testing, plasma and serum samples were heat-inactivated at $56^{\circ} \mathrm{C}$ for 1 hour. Plates were read at an optical density (OD) of 450nm using the SpectraMax 190 microplate reader (Molecular Devices). Background OD values from the plates coated with PBS were subtracted from the OD values from plates coated with recombinant protein. Each plate included serial dilutions of the IgG monoclonal antibody CR3022, which is reactive to the SARS-CoV-2 spike protein, as a positive control to 
adjust for inter assay variability. Plasma and serum antibody concentrations were reported as arbitrary units relative to the CR3022 monoclonal antibody. A cutoff of 0.48 arbitrary units was established from a 2019 cohort of pre-pandemic individuals and used for defining seropositivity.

\section{Flow Cytometry and statistical analysis}

Samples were acquired on a 5 laser BD FACS Symphony A5. Standardized SPHERO rainbow beads (Spherotech, Cat\#RFP-30-5A) were used to track and adjust PMTs over time. UltraComp eBeads (ThermoFisher, Cat\#01-2222-42) were used for compensation. Up to $2 \times$ $10^{\wedge} 6$ live PBMC were acquired per each sample. During the early sample acquisition period, three antibodies in the flow panel were changed. Three cancer patients and twelve non-cancer patients were stained using this earlier flow panel. Flow features of these patients were visually assessed for batch variations against data from the later flow panel. The three cancer patients were included with the rest of the cohort when batch effects were determined to have little impact on confidence in gated populations. These three cancer patients were excluded in analysis of cell populations defined by proteins associated with the three changed antibodies.

Due to the heterogeneity of clinical and flow cytometric data, non-parametric tests of association were preferentially used throughout the study. Tests of association between mixed continuous variables versus non-ordered categorical variables $(n=2)$ were performed by MannWhitney test. Tests of association between binary variables versus non-ordered categorical variables $(n=2)$ were performed using Pearson Chi Square test. All tests were performed using a nominal significance threshold of $\mathrm{P}<0.05$ with Prism version 9 (GraphPad Software) and Excel (Microsoft Office Suite). Classification and Regression Tree analysis (CART) was performed using R package 'rpart'. 
UMAP analyses were conducted using R package uwot. FlowSOM analyses were

552 performed on Cytobank (https://cytobank.org). Lymphocytes and non-naive CD8 T cells were

553 analyzed separately. An artifact due to monocyte contamination was removed from the FCS as

554 defined by high CD16 and side scatter area (SSC-A). UMAP analysis was performed using

555 equal down sampling of 10000 cells from each FCS file in lymphocytes and 1500 cells in non-

556 naive CD8 T cells, with a nearest neighbors of 15 , minimum distance of 0.01 , number of

557 components of 2, and a euclidean metric. The FCS files were then fed into the FlowSOM

558 clustering algorithm. A new self-organizing map (SOM) was generated for both lymphocytes and

559 non-naive CD8 using hierarchical consensus clustering. For each SOM, 225 clusters and 10

560 metaclusters were identified. For lymphocytes, the following markers were used in the UMAP

561 and FlowSOM analysis: CD45RA, PD-1, IgD, CXCR5, CD8, CD19, CD3, CD16, CD138,

562 Eomes, TCF-1, CD38, CD95, CCR7, CD21, Ki-67, CD27, CD4, CX3CR1, CD39, T-bet, HLA-

563 DR, and CD20. For non-naive CD8 T cells, the following markers were used: CD45RA, PD-1,

564 CXCR5, CD16, Eomes, TCF-1, CD38, CCR7, Ki-67, CD27, CX3CR1, CD39, T-bet, and HLA-

565 DR. For FlowSOM analysis of non-naive CD8 T cells, two patients at day seven without data

566 from day zero were included. Heatmaps were visualized using R function pheatmap.

567 To group individuals based on lymphocyte landscape, pairwise Earth Mover's Distance

568 (EMD) value was calculated on the lymphocyte UMAP axes using the emdist package in R.

569 Resulting scores were hierarchically clustered using the hclust package in R.

\section{Immune profiling of patients hospitalized for COVID-19, MSKCC}

572 Patients admitted to Memorial Sloan Kettering Cancer Center with a positive SARS-CoV-2 PCR

573 test were eligible for inclusion. For inpatients, clinical data were abstracted from the electronic

574 medical record into standardized case report forms. Clinical laboratory data were abstracted

575 from the date closest to research blood collection. Peripheral blood was collected into BD

576 Horizon Dri tubes (BD, Cat\#625642). Immunophenotyping of peripheral blood mononuclear 
cells via flow cytometry was performed in the MSKCC clinical laboratory. The lymphocyte panel

578 included CD45 FITC (BD, 340664, clone 2D1), CD56+16 PE (BD 340705, clone B73.1; BD

579 340724, clone NCAM 16.2), CD4 PerCP-Cy5.5 (BD 341653, clone SK3), CD45RA PC7 (BD

580 649457, clone L48), CD19 APC (BD 340722, clone SJ25C1), CD8 APC-H7 (BD 641409, clone

581 SK1), and CD3 BV 421 (BD 562426, clone UCHT1). The naive/effector T panel included CD45

582 FITC (BD 340664, clone 2D1), CCR7 PE (BD 560765, clone 150503), CD4 PerCP-Cy5.5 (BD

583 341653, clone SK3), CD38 APC (BioLegend, 303510, clone HIT2), HLA-DR V500 (BD 561224,

584 clone G46-6), CD45RA PC7 (BD 649457, clone L48), CD8 APC-H7 (BD 641409, clone SK1),

585 and CD3 BV 421 (BD 562426, clone UCHT1). The immune phenotypes were based on NIH

586 vaccine consensus panels and the Human Immunology Project ${ }^{74}$. Samples were acquired on a

BD Facs Canto using FACSDiva software.

Data Availability Statement: Flow Cytometry data collected in this study was deposited to the Human Pancreas Analysis Program (HPAP-RRID:SCR_016202) Database and Cytobank62

\section{Funding}

ACH was funded by grant CA230157 from the NIH. NJM was supported by NIH HL137006, HL137915. DM was funded by T32 CA009140. JRG is a Cancer Research Institute-Mark Foundation Fellow. ALG was supported by the Leukemia and Lymphoma Society Scholar in Clinical Research Award. JRG, JEW, CA, ACH, and EJW are supported by the Parker Institute for Cancer Immunotherapy which supports the Cancer Immunology program at the University of Pennsylvania. E.J.W. was supported by NIH grants Al155577, Al112521, Al082630, Al201085, Al123539, Al117950 and funding from the Allen Institute for Immunology to E.J.W. SV is supported by funding from the Pershing Square Sohn Cancer Research Foundation. SV is a consultant from Immunai and ADC therapeutics. ACH is a consultant for Immunai. RHV reports having received consulting fees from Medimmune and Verastem; and research funding from Fibrogen, Janssen, and Lilly. He is an inventor on a licensed patents relating to cancer cellular immunotherapy and cancer vaccines, and receives royalties from Children's Hospital Boston for a licensed research-only monoclonal antibody. JW is serving as a consultant for Adaptive Biotechnologies, Advaxis, Amgen, Apricity, Array BioPharma, Ascentage Pharma, Astellas, Bayer, BeiGene, Bristol-Myers Squibb. Celgene, Chugai, Elucida, Eli Lilly, F-Star, Genentech, Imvaq, Janssen, Kyowa Hakko Kirin, Kleo Pharmaceuticals, Linnaeus, Medlmmune, Merck, Neon Therapeutics, Northern Biologics, Ono, Polaris Pharma, Polynoma, PsiOxus, PureTech, Recepta, Takara Bio, Trieza, Sellas Life Sciences, Serametrix, Surface Oncology, Syndax and Synthologic. JW received research support from Bristol-Myers Squibb, Medlmmune, Merck and Genentech and has equity in Potenza Therapeutics, Tizona Pharmaceuticals, Adaptive 


\section{Author contributions}

$\mathrm{ACH}, \mathrm{RM}, \mathrm{SV}$, and EMB conceived the project; $\mathrm{ACH}, \mathrm{SV}, \mathrm{NAH}$ designed all experiments. $\mathrm{ACH}$, $\mathrm{RM}, \mathrm{EMB}, \mathrm{AMD}$, IPM conceived the PENN COPE cohort. EMB, JR, FP, OO, KN, CZ, MG, ARW, CAGI, EMK, CR, KRB, ST, and CW enrolled patients and collected data for COPE. EMB, $\mathrm{RM}, \mathrm{AMD}$, and $\mathrm{ACH}$ designed data and statistical analysis for COPE. PW performed statistical analysis for COPE. NJM conceived the PENN MESSI-COVID clinical cohort, ARW, CAGI, OO, RSA, TGD, TJ, HMG, JPR, and NJM enrolled patients and collected data for MESSI-

COVID. NAH, AEB, and JYK performed downstream flow cytometry analysis for MESSICOVID. SG, MEW, CMM, SEH analyzed COVID-19 patient plasma and provided antibody data. $\mathrm{NAH}, \mathrm{JRG}, \mathrm{ARG}, \mathrm{CA}$, DAO performed computational and statistical analyses. CA compiled and JRG, DO, and CA analyzed clinical metadata for MESSI-COVID.

SV and JW conceived the MSKCC cohort. SV, AK, AW, SD, and SB provided clinical samples from MSKCC Cohort. PM performed downstream flow cytometry analysis. NEB performed quantitative PCR experiments for COVID viral load. NAH and ACH compiled figures. KNM, LS, RHV, JDW, EJW, provided intellectual input. EMB, SV, RM, and ACH wrote the manuscript; all authors review the manuscript.

\section{Acknowledgements}

The authors thank patients and blood donors, their families and surrogates, and medical personnel. In addition we thank the UPenn COVID Processing Unit: A unit of individuals from diverse laboratories at the University of Pennsylvania who volunteered time and effort to enable study of COVID-19 patients during the pandemic: Sharon Adamski, Zahidul Alam, Mary M. Addison, Katelyn T. Byrne, Aditi Chandra, Hélène C. Descamps, Nicholas Han, Yaroslav Kaminskiy, Shane C. Kammerman, Justin Kim, Allison R. Greenplate, Kurt D'Andrea, Jacob T. Hamilton, Nune Markosyan, Julia Han Noll, Dalia K. Omran, Ajinkya Pattekar, Eric Perkey, Elizabeth M. Prager, Dana Pueschl, Austin Rennels, Jennifer B. Shah, Jake S. Shilan, Nils Wilhausen, Ashley N. Vanderbeck. All affiliated with the University of Pennsylvania Perelman School of Medicine. 


\section{References}

659 1. Prescott HC, Girard TD: Recovery From Severe COVID-19: Leveraging the Lessons of Survival From 660 Sepsis. JAMA 324:739, 2020

661 2. Wu Z, McGoogan JM: Characteristics of and Important Lessons From the Coronavirus Disease 2019 662 (COVID-19) Outbreak in China: Summary of a Report of 72314 Cases From the Chinese Center for 663 Disease Control and Prevention [Internet]. JAMA , 2020[cited 2020 Mar 28] Available from:

664 https://jamanetwork.com/journals/jama/fullarticle/2762130

665 3. Blanco-Melo D, Nilsson-Payant BE, Liu W-C, et al: Imbalanced Host Response to SARS-CoV-2 Drives 666 Development of COVID-19. Cell 181:1036-1045.e9, 2020

667 4. Hadjadj J, Yatim N, Barnabei L, et al: Impaired type I interferon activity and inflammatory responses in 668 severe COVID-19 patients. Science 369:718-724, 2020

669 5. Arunachalam PS, Wimmers F, Mok CKP, et al: Systems biological assessment of immunity to mild 670 versus severe COVID-19 infection in humans. Science 369:1210-1220, 2020

671 6. Chen G, Wu D, Guo W, et al: Clinical and immunological features of severe and moderate coronavirus 672 disease 2019. J Clin Invest 130:2620-2629, 2020

673 7. Huang C, Wang Y, Li X, et al: Clinical features of patients infected with 2019 novel coronavirus in 674 Wuhan, China. The Lancet 395:497-506, 2020

675 8. Tan L, Wang Q, Zhang D, et al: Lymphopenia predicts disease severity of COVID-19: a descriptive and 676 predictive study [Internet]. Signal Transduct Target Ther 5, 2020[cited 2020 Oct 20] Available from: 677 http://www.nature.com/articles/s41392-020-0148-4

678 9. Zhao Q, Meng M, Kumar R, et al: Lymphopenia is associated with severe coronavirus disease 2019 679 (COVID-19) infections: A systemic review and meta-analysis. Int J Infect Dis 96:131-135, 2020

680 10. Qin C, Zhou L, Hu Z, et al: Dysregulation of Immune Response in Patients With Coronavirus 2019 681 (COVID-19) in Wuhan, China. Clin Infect Dis 71:762-768, 2020

682 11. Laing AG, Lorenc A, del Molino del Barrio I, et al: A dynamic COVID-19 immune signature includes 683 associations with poor prognosis. Nat Med 26:1623-1635, 2020

684 12. Yale IMPACT Team, Lucas $C$, Wong $P$, et al: Longitudinal analyses reveal immunological misfiring in 685 severe COVID-19. Nature 584:463-469, 2020

686 13. Giamarellos-Bourboulis EJ, Netea MG, Rovina N, et al: Complex Immune Dysregulation in COVID-19 687 Patients with Severe Respiratory Failure. Cell Host Microbe 27:992-1000.e3, 2020

688 14. Mann ER, Menon M, Knight SB, et al: Longitudinal immune profiling reveals key myeloid signatures 689 associated with COVID-19. Sci Immunol 5:eabd6197, 2020

690 15. Mathew D, Giles JR, Baxter AE, et al: Deep immune profiling of COVID-19 patients reveals distinct 691 immunotypes with therapeutic implications. Science 369:eabc8511, 2020 
16. Su Y, Chen D, Yuan D, et al: Multi-Omics Resolves a Sharp Disease-State Shift between Mild and Moderate COVID-19. Cell 183:1479-1495.e20, 2020

17. De Biasi S, Meschiari M, Gibellini L, et al: Marked T cell activation, senescence, exhaustion and skewing towards TH17 in patients with COVID-19 pneumonia [Internet]. Nat Commun 11, 2020[cited 2020 Dec 22] Available from: http://www.nature.com/articles/s41467-020-17292-4

18. Zheng $\mathrm{H}-\mathrm{Y}$, Zhang $\mathrm{M}$, Yang $\mathrm{C}-\mathrm{X}$, et al: Elevated exhaustion levels and reduced functional diversity of $\mathrm{T}$ cells in peripheral blood may predict severe progression in COVID-19 patients. Cell Mol Immunol 17:541-543, 2020

19. Xu Z, Shi L, Wang Y, et al: Pathological findings of COVID-19 associated with acute respiratory distress syndrome. Lancet Respir Med , 2020

20. Kuri-Cervantes $L$, Pampena $M B$, Meng $W$, et al: Comprehensive mapping of immune perturbations associated with severe COVID-19. Sci Immunol 5:eabd7114, 2020

21. Zhao J, Yuan $\mathrm{Q}$, Wang $\mathrm{H}$, et al: Antibody Responses to SARS-CoV-2 in Patients With Novel Coronavirus Disease 2019. Clin Infect Dis 71:2027-2034, 2020

22. Long Q-X, Liu B-Z, Deng H-J, et al: Antibody responses to SARS-CoV-2 in patients with COVID-19. Nat Med 26:845-848, 2020

23. Rugge $M$, Zorzi $M$, Guzzinati S: SARS-CoV-2 infection in the Italian Veneto region: adverse outcomes in patients with cancer. Nat Cancer 1:784-788, 2020

24. Assaad S, Avrillon V, Fournier M-L, et al: High mortality rate in cancer patients with symptoms of COVID-19 with or without detectable SARS-COV-2 on RT-PCR. Eur J Cancer 135:251-259, 2020

25. Miyashita H, Kuno T: Prognosis of coronavirus disease 2019 (COVID-19) in patients with HIV infection in New York City [Internet]. HIV Med, 2020[cited 2020 Oct 20] Available from: https://onlinelibrary.wiley.com/doi/abs/10.1111/hiv.12920

26. Dai M, Liu D, Liu M, et al: Patients with cancer appear more vulnerable to SARS-COV-2: a multicenter study during the COVID-19 outbreak. Cancer Discov CD-20-0422, 2020

27. Saini KS, Tagliamento M, Lambertini M, et al: Mortality in patients with cancer and coronavirus disease 2019: A systematic review and pooled analysis of 52 studies. Eur J Cancer Oxf Engl 1990 139:4350,2020

28. Coronavirus COVID-19 Global Cases [Internet], 2020[cited 2020 Oct 19] Available from: https://coronavirus.jhu.edu/map.html

29. Mehta V, Goel S, Kabarriti R, et al: Case Fatality Rate of Cancer Patients with COVID-19 in a New York Hospital System. Cancer Discov CD-20-0516, 2020

30. Lee LYW, Cazier J-B, Starkey T, et al: COVID-19 prevalence and mortality in patients with cancer and the effect of primary tumour subtype and patient demographics: a prospective cohort study. Lancet Oncol 21:1309-1316, 2020 
31. Mato AR, Roeker LE, Lamanna N, et al: Outcomes of COVID-19 in patients with CLL: a multicenter international experience. Blood 136:1134-1143, 2020

32. Chari A, Samur MK, Martinez-Lopez J, et al: Clinical features associated with COVID-19 outcome in multiple myeloma: first results from the International Myeloma Society data set. Blood 136:3033-3040, 2020

33. Lamure S, Duléry R, Di Blasi R, et al: Determinants of outcome in Covid-19 hospitalized patients with lymphoma: A retrospective multicentric cohort study. EClinicalMedicine 27:100549, 2020

34. Lee LYW, Cazier JB, Starkey T, et al: COVID-19 mortality in patients with cancer on chemotherapy or other anticancer treatments: a prospective cohort study [Internet]. The Lancet , 2020[cited 2020 Jun 7] Available from: https://linkinghub.elsevier.com/retrieve/pii/S0140673620311739

35. Albiges L, Foulon S, Bayle A, et al: Determinants of the outcomes of patients with cancer infected with SARS-CoV-2: results from the Gustave Roussy cohort [Internet]. Nat Cancer , 2020[cited 2020 Oct 19] Available from: http://www.nature.com/articles/s43018-020-00120-5

36. Kuderer NM, Choueiri TK, Shah DP, et al: Clinical impact of COVID-19 on patients with cancer (CCC19): a cohort study [Internet]. The Lancet , 2020[cited 2020 Jun 7] Available from: https://linkinghub.elsevier.com/retrieve/pii/S0140673620311879

37. Garassino MC, Whisenant JG, Huang L-C, et al: COVID-19 in patients with thoracic malignancies (TERAVOLT): first results of an international, registry-based, cohort study. Lancet Oncol 21:914-922, 2020

38. Petrilli CM, Jones SA, Yang J, et al: Factors associated with hospital admission and critical illness among 5279 people with coronavirus disease 2019 in New York City: prospective cohort study. BMJ 369:m1966, 2020

39. Williamson EJ, Walker AJ, Bhaskaran K, et al: Factors associated with COVID-19-related death using OpenSAFELY. Nature 584:430-436, 2020

40. Zhou F, Yu T, Du R, et al: Clinical course and risk factors for mortality of adult inpatients with COVID19 in Wuhan, China: a retrospective cohort study. The Lancet 395:1054-1062, 2020

41. Jee J, Foote MB, Lumish M, et al: Chemotherapy and COVID-19 Outcomes in Patients With Cancer. J Clin Oncol 38:3538-3546, 2020

42. Robilotti EV, Babady NE, Mead PA, et al: Determinants of COVID-19 disease severity in patients with cancer. Nat Med 26:1218-1223, 2020

43. Amanat F, Stadlbauer D, Strohmeier S, et al: A serological assay to detect SARS-CoV-2 seroconversion in humans. Nat Med 26:1033-1036, 2020

44. Flannery DD, Gouma S, Dhudasia MB, et al: SARS-CoV-2 seroprevalence among parturient women in Philadelphia. Sci Immunol 5:eabd5709, 2020

45. Orlova DY, Zimmerman N, Meehan S, et al: Earth Mover's Distance (EMD): A True Metric for Comparing Biomarker Expression Levels in Cell Populations. PLOS ONE 11:e0151859, 2016 
46. Del Valle DM, Kim-Schulze $\mathrm{S}$, Huang $\mathrm{H}-\mathrm{H}$, et al: An inflammatory cytokine signature predicts COVID19 severity and survival. Nat Med 26:1636-1643, 2020

47. RECOVERY Collaborative Group, Horby P, Lim WS, et al: Dexamethasone in Hospitalized Patients with Covid-19 - Preliminary Report. N Engl J Med , 2020

48. Shields AM, Burns SO, Savic S, et al: COVID-19 in patients with primary and secondary immunodeficiency: the United Kingdom experience. J Allergy Clin Immunol , 2020

49. Gianfrancesco M, Hyrich KL, Al-Adely S, et al: Characteristics associated with hospitalisation for COVID-19 in people with rheumatic disease: data from the COVID-19 Global Rheumatology Alliance physician-reported registry. Ann Rheum Dis 79:859-866, 2020

50. Pereira MR, Mohan S, Cohen DJ, et al: COVID-19 in solid organ transplant recipients: Initial report from the US epicenter. Am J Transplant Off J Am Soc Transplant Am Soc Transpl Surg 20:1800-1808, 2020

51. Raja MA, Mendoza MA, Villavicencio A, et al: COVID-19 in solid organ transplant recipients: $A$ systematic review and meta-analysis of current literature. Transplant Rev Orlando Fla 35:100588, 2020

52. Thevarajan I, Nguyen THO, Koutsakos M, et al: Breadth of concomitant immune responses prior to patient recovery: a case report of non-severe COVID-19. Nat Med 26:453-455, 2020

53. Vijenthira A, Gong IY, Fox TA, et al: Outcomes of patients with hematologic malignancies and COVID19: A systematic review and meta-analysis of 3377 patients. Blood , 2020

54. Persad G, Peek ME, Emanuel EJ: Fairly Prioritizing Groups for Access to COVID-19 Vaccines. JAMA, 2020

55. Zhang B, Zhou X, Zhu C, et al: Immune Phenotyping Based on the Neutrophil-to-Lymphocyte Ratio and IgG Level Predicts Disease Severity and Outcome for Patients With COVID-19. Front Mol Biosci 7:157, 2020

56. Liu J, Liu Y, Xiang P, et al: Neutrophil-to-lymphocyte ratio predicts critical illness patients with 2019 coronavirus disease in the early stage. J Transl Med 18:206, 2020

57. Bastard P, Rosen LB, Zhang Q, et al: Autoantibodies against type I IFNs in patients with lifethreatening COVID-19. Science 370, 2020

58. Abdul-Jawad S, Baù L, Alaguthurai $T$, et al: Acute immune signatures and their legacies in severe acute respiratory syndrome coronavirus- 2 infected cancer patients. Cancer Cell S1535610821000015, 2021

59. Rydyznski Moderbacher C, Ramirez SI, Dan JM, et al: Antigen-Specific Adaptive Immunity to SARSCoV-2 in Acute COVID-19 and Associations with Age and Disease Severity. Cell 183:996-1012.e19, 2020

60. Weidt G, Utermöhlen O, Zerrahn J, et al: CD8+ T lymphocyte-mediated antiviral immunity in mice as a result of injection of recombinant viral proteins. J Immunol Baltim Md 1950 153:2554-2561, 1994

61. Sun J, Zhuang Z, Zheng J, et al: Generation of a Broadly Useful Model for COVID-19 Pathogenesis, Vaccination, and Treatment. Cell 182:734-743.e5, 2020 
823

824

825

826

827

62. Sekine T, Perez-Potti A, Rivera-Ballesteros O, et al: Robust T Cell Immunity in Convalescent Individuals with Asymptomatic or Mild COVID-19. Cell 183:158-168.e14, 2020

63. Peng $Y$, Mentzer AJ, Liu G, et al: Broad and strong memory CD4+ and CD8+ T cells induced by SARSCoV-2 in UK convalescent individuals following COVID-19. Nat Immunol 21:1336-1345, 2020

64. Kared H, Redd AD, Bloch EM, et al: SARS-CoV-2-specific CD8+ T cell responses in convalescent COVID19 individuals. J Clin Invest , 2021

65. Ni L, Ye F, Cheng M-L, et al: Detection of SARS-CoV-2-Specific Humoral and Cellular Immunity in COVID-19 Convalescent Individuals. Immunity 52:971-977.e3, 2020

66. Cook AM, McDonnell AM, Lake RA, et al: Dexamethasone co-medication in cancer patients undergoing chemotherapy causes substantial immunomodulatory effects with implications for chemoimmunotherapy strategies. Oncoimmunology 5:e1066062, 2016

67. Takeda S, Rodewald H-R, Arakawa H, et al: MHC Class II Molecules Are Not Required for Survival of Newly Generated CD4+ T Cells, but Affect Their Long-Term Life Span. Immunity 5:217-228, 1996

68. Stefanová I, Dorfman JR, Germain RN: Self-recognition promotes the foreign antigen sensitivity of naive T lymphocytes. Nature 420:429-434, 2002

69. Sadoff J, Le Gars M, Shukarev G, et al: Interim Results of a Phase 1-2a Trial of Ad26.COV2.S Covid-19 Vaccine. N Engl J Med , 2021

70. Corbett KS, Edwards DK, Leist SR, et al: SARS-CoV-2 mRNA vaccine design enabled by prototype pathogen preparedness. Nature 586:567-571, 2020

71. Sahin U, Muik A, Derhovanessian E, et al: COVID-19 vaccine BNT162b1 elicits human antibody and TH1 T cell responses. Nature 586:594-599, 2020

72. Global surveillance for COVID-19 caused by human infection with COVID-19 virus. [Internet]. WHO, 2020Available from: https://apps.who.int/iris/bitstream/handle/10665/331506/WHO-2019-nCoVSurveillanceGuidance-2020.6-eng.pdf

73. Beigel JH, Tomashek KM, Dodd LE, et al: Remdesivir for the Treatment of Covid-19 - Final Report. N Engl J Med 383:1813-1826, 2020

74. Maecker HT, McCoy JP, Nussenblatt R: Standardizing immunophenotyping for the Human Immunology Project. Nat Rev Immunol 12:191-200, 2012 
Table 1 | COPE: Patient demographics and clinical characteristics.

\begin{tabular}{|c|c|}
\hline & Total $(N=100)$ \\
\hline Age, median (IQR) & $68(57.5-77.5)$ \\
\hline Gender, female & $48(48 \%)$ \\
\hline \multicolumn{2}{|l|}{ Race } \\
\hline Black & $54(54 \%)$ \\
\hline White & $33(33 \%)$ \\
\hline Asian & $4(4 \%)$ \\
\hline Hispanic & $3(3 \%)$ \\
\hline Unknown & $6(6 \%)$ \\
\hline Smoking History, Ever ${ }^{+}$ & $57(57 \%)$ \\
\hline \multicolumn{2}{|l|}{ Comorbidities } \\
\hline Cardiac & $78(78 \%)$ \\
\hline Pulmonary & $41(41 \%)$ \\
\hline Use of immunosuppressive drugs ${ }^{++}$ & $30(30 \%)$ \\
\hline BMI, median (IQR) & $26.84(23.2-31.5)$ \\
\hline \multicolumn{2}{|l|}{ Cancer Type } \\
\hline Solid malignancy & $78(78 \%)$ \\
\hline Genitourinary & $19(19 \%)$ \\
\hline Breast & $14(14 \%)$ \\
\hline Gastrointestinal & $14(14 \%)$ \\
\hline Thoracic & $9(9 \%)$ \\
\hline Other ${ }^{+++}$ & $8(8 \%)$ \\
\hline Gynecologic & $7(7 \%)$ \\
\hline Head and Neck & $4(4 \%)$ \\
\hline Sarcoma & $3(3 \%)$ \\
\hline Heme malignancy & $22(22 \%)$ \\
\hline Lymphoma & $10(10 \%)$ \\
\hline Leukemia & $7(7 \%)$ \\
\hline Myeloma & $3(3 \%)$ \\
\hline MDS/MPN & $2(2 \%)$ \\
\hline Cancer Status, Active ${ }^{\#}$ & $46(46 \%)$ \\
\hline \multicolumn{2}{|l|}{ Cancer treatment in last 3 months } \\
\hline Active surveillance/surgery & $53(53 \%)$ \\
\hline Cytotoxic Chemotherapy & $24(24 \%)$ \\
\hline Hormone therapy & $15(15 \%)$ \\
\hline Other* & $8(8 \%)$ \\
\hline ECOG Performance Status & $\mathrm{N}=73$ \\
\hline $0-1$ & $37(50.7 \%)$ \\
\hline 2 & $13(17.8 \%)$ \\
\hline $3-4$ & $23(31.5 \%)$ \\
\hline \multicolumn{2}{|c|}{$\begin{array}{l}{ }^{+} \text {Current or prior smoker } \\
{ }^{++} \text {Exposure to immunosuppressive medications not including cancer treatment } \\
+++ \text { Tumor types with less than } 2 \text { subjects: CNS-2, Thyroid-2, Thymus-1, Neuroendocrine-1 } \\
\text { "Diagnosis or treatment within } 6 \text { months } \\
\text { "Single agent immunotherapy, targeted therapy, monoclonal antibodies }\end{array}$} \\
\hline
\end{tabular}


Table 2 | COPE: COVID-19 related treatment and outcomes.

\begin{tabular}{|c|c|c|c|}
\hline \multicolumn{2}{|c|}{ Total (N=100) } & \multicolumn{1}{l|}{ Solid (N=78) } & \\
\hline COVID-19 Disease Severity & & & \\
\hline At Presentation & & $28(35.9 \%)$ & $7(31.85)$ \\
\hline No Supplemental Oxygen & $35(35.0 \%)$ & $32(41.0 \%)$ & $12(54.6 \%)$ \\
\hline Supplemental Oxygen & $44(44.0 \%)$ & $7(8.97 \%)$ & $2(9.09 \%)$ \\
\hline Non-invasive ventilation & $9(9.00 \%)$ & $11(14.1 \%)$ & $1(4.55 \%)$ \\
\hline Invasive ventilation & $12(12.0 \%)$ & & \\
\hline Maximum throughout hospitalization & & $24(30.8 \%)$ & $4(18.2 \%)$ \\
\hline No supplemental Oxygen & $28(28.0 \%)$ & $19(24.4 \%)$ & $4(18.2 \%)$ \\
\hline Supplemental Oxygen & $24(24.0 \%)$ & $8(10.3 \%)$ & $3(13.6 \%)$ \\
\hline Non-invasive ventilation & $11(11.0 \%)$ & $9(11.5 \%)$ & $0(0.00 \%)$ \\
\hline Invasive ventilation & $9(9.00 \%)$ & $18(23.1 \%)$ & $12(54.5 \%)$ \\
\hline Death & $28(28.0 \%)$ & & \\
\hline COVID-19 Directed Treatment & & $39(50.0 \%)$ & $12(54.6 \%)$ \\
\hline Steroids & $51(51.0 \%)$ & $13(16.7 \%)$ & $5(22.7 \%)$ \\
\hline Remdesivir & $18(18.0 \%)$ & $6(7.69 \%)$ & $4(18.2 \%)$ \\
\hline Convalescent Plasma & $10(10.0 \%)$ & & \\
\hline COVID-19 Outcomes & & $7(9.09 \%)$ & $4(18.2 \%)$ \\
\hline Thrombosis & $11(11.0 \%)$ & $21(26.9 \%)$ & $7(31.8 \%)$ \\
\hline Intubation & $28(28.0 \%)$ & $37(47.4 \%)$ & $11(50.0 \%)$ \\
\hline ICU admission & $48(48.0 \%)$ & $26(33.3 \%)$ & $12(54.6 \%)$ \\
\hline Death & $38(38.0 \%)$ & $8(4-18)$ \\
\hline Hospital Length of stay, median (IQR) & & & \\
\hline
\end{tabular}


Table 3 | COPE: Event rates and point estimates of outcomes by cancer type.

\begin{tabular}{|c|c|c|}
\hline & Heme & Solid \\
\hline \multicolumn{3}{|c|}{ Death within 30 days of discharge } \\
\hline Event rate (\%) & $12(54.6 \%)$ & $26(33.3 \%)$ \\
\hline Unadjusted OR (95\% Cl) & $2.4(0.82-7.06)$ & ref \\
\hline Adjusted OR $(95 \% \mathrm{Cl})^{+}$ & $3.3(1.01-10.8)$ & ref \\
\hline Adjusted HR $(95 \% \mathrm{Cl})^{+}$ & $2.6(1.19-5.54)$ & ref \\
\hline
\end{tabular}


Fig. 1

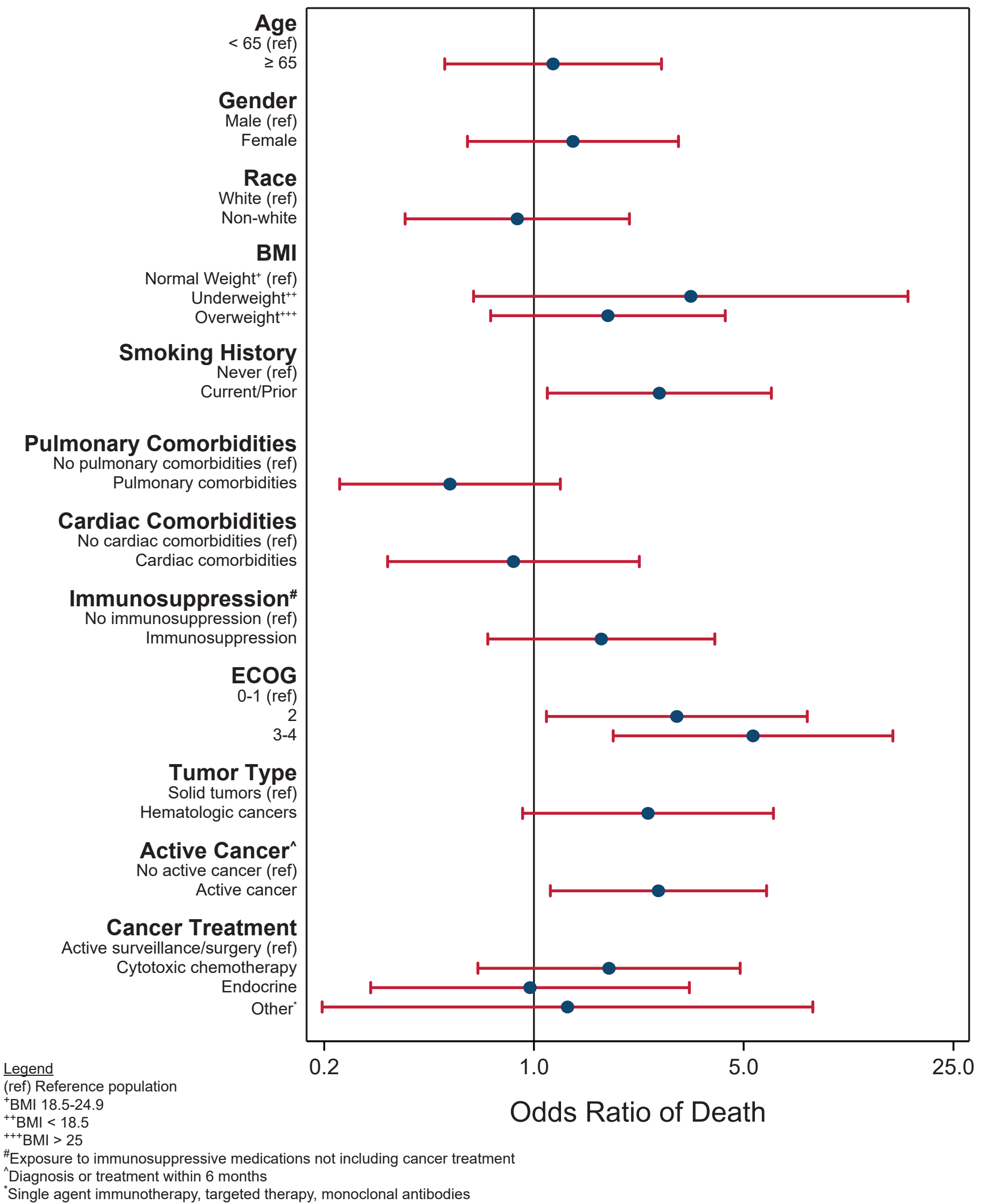

Fig. 1 | Univariate analysis of potential risk factors in COVID-19 mortality. 
Fig. 2
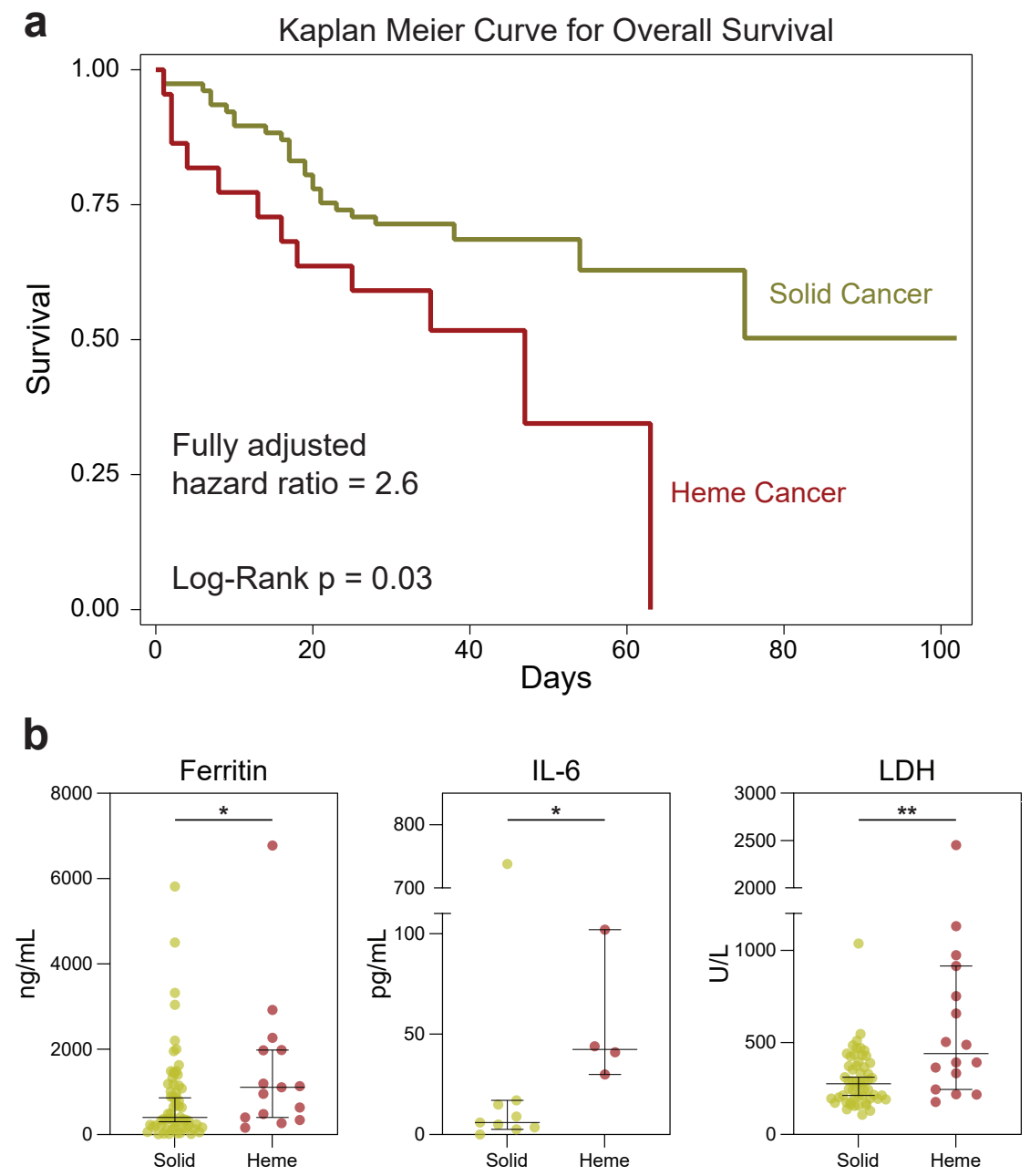

Fig. 2 | Hematologic cancer is an independent risk factor for COVID-19 related mortality. (a) Kaplan Meier curve for COVID-19 survival of patients with solid ( $n=77$ ) and hematologic $(n=22)$ cancer. Cox regression-computed hazard ratio for mortality in hematologic vs solid cancer, adjusted for age, gender, smoking status, active cancer status, and ECOG performance status. (b) Ferritin, IL-6, and LDH in solid $(n=62)$ and hematologic $(n=15)$ cancer hospitalized for COVID-19. (All) Significance determined by Mann Whitney test: ${ }^{*} p<0.05,{ }^{* *} p<0.01,{ }^{* * *} p<0.001$, and ${ }^{* * * *} p<0.0001$. Median and $95 \%$ Cl shown. 
Fig. 3

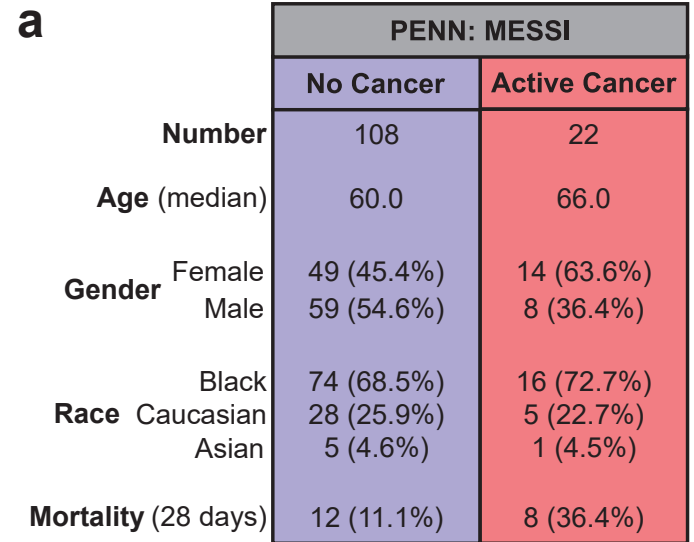

e

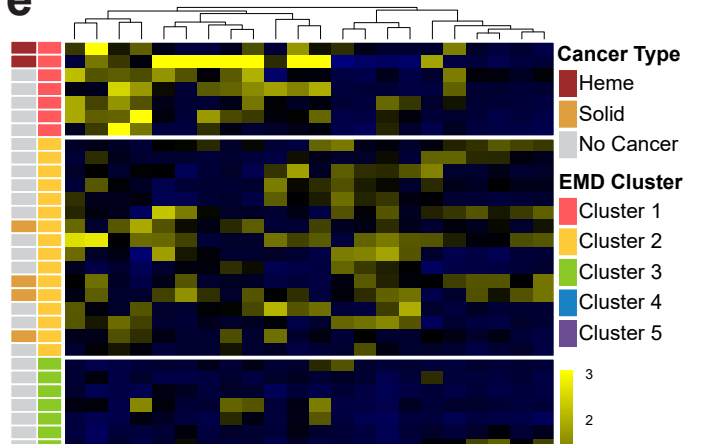

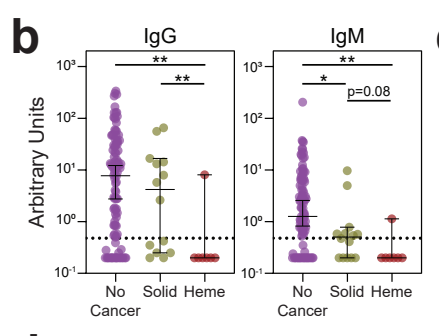
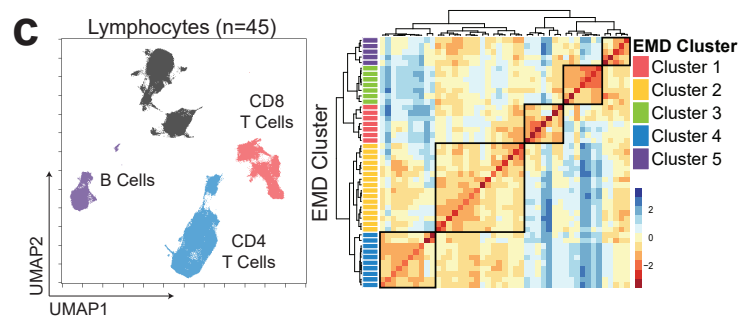

d
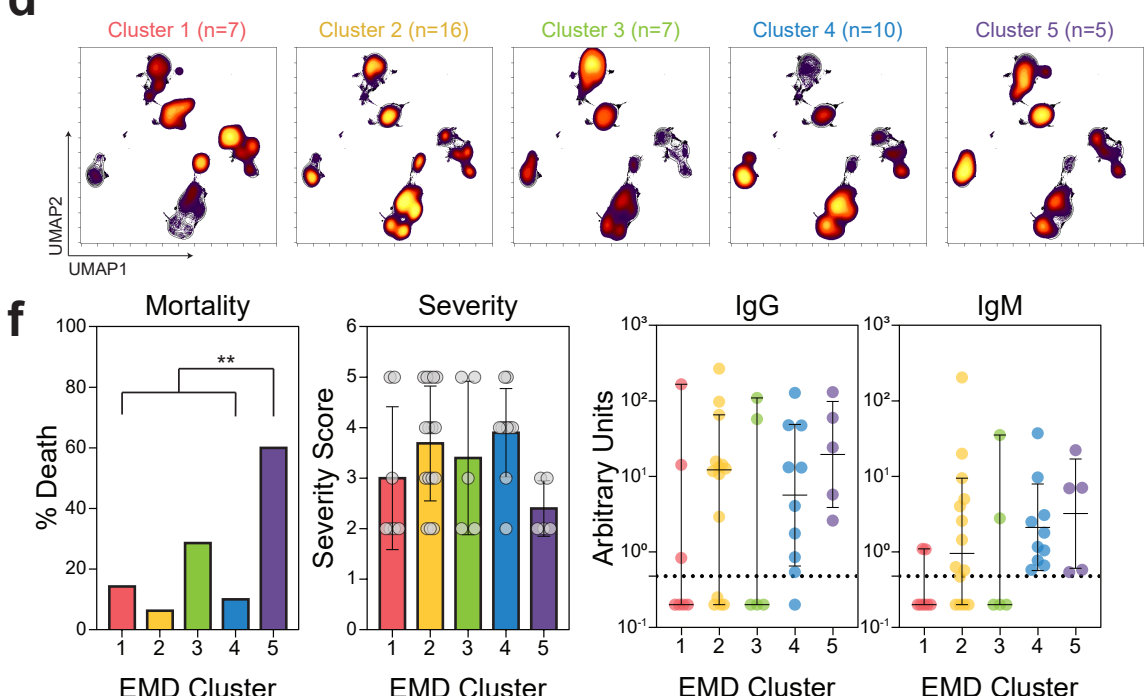
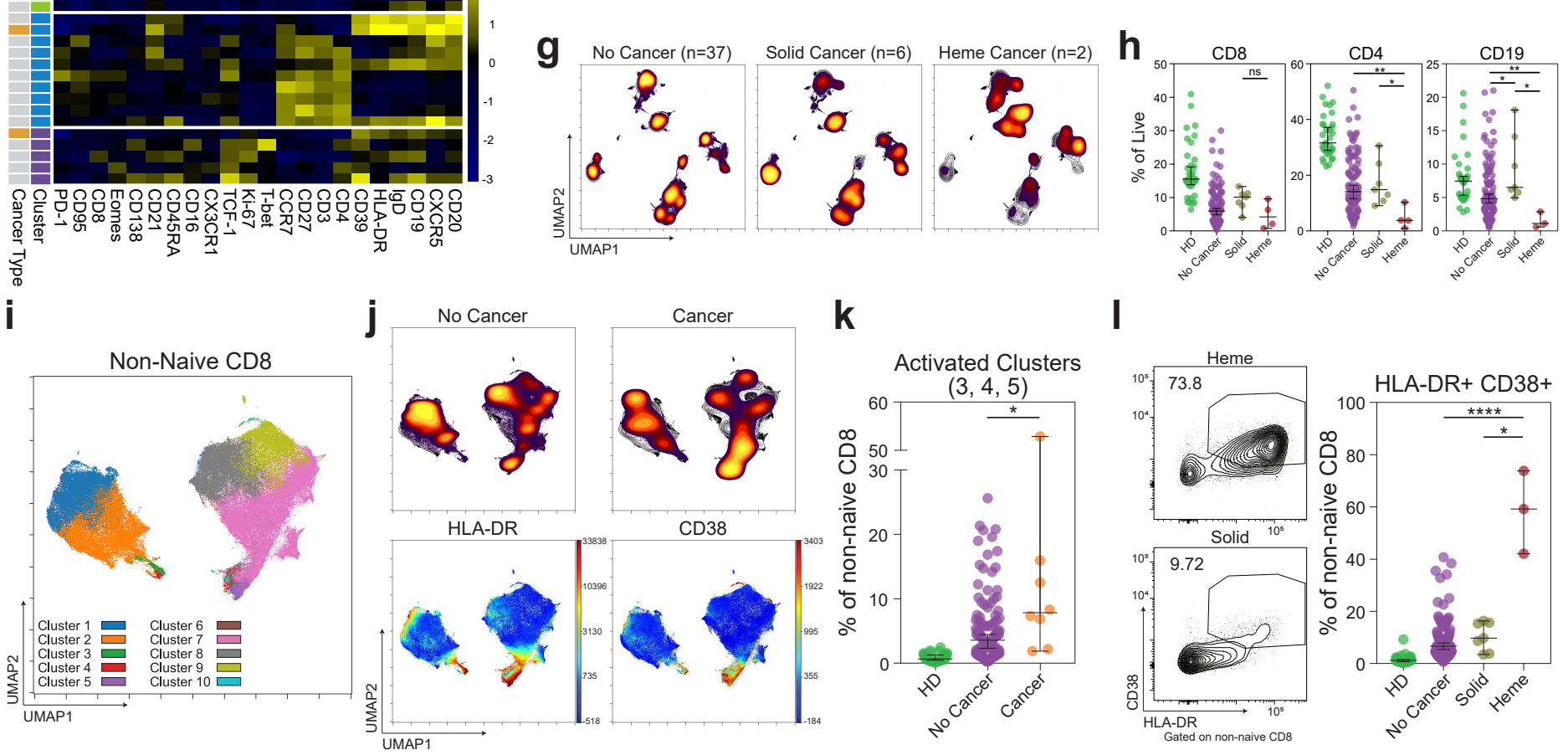

$\mathbf{k}$
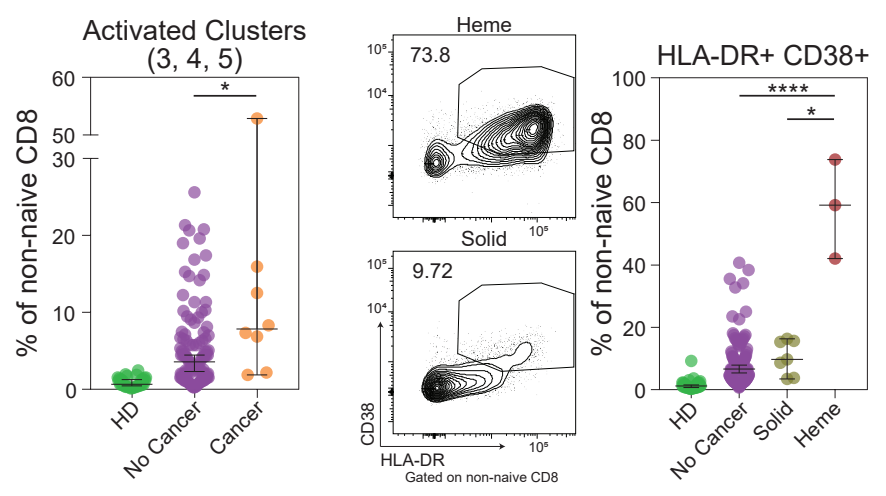

Fig. 3 | High dimensional analyses reveal immune phenotypes associated with mortality and distinct phenotypes between solid and hematologic cancers. (a) Demographic and mortality data for MESSI cohort at Penn. (b) Relative levels of SARS-CoV-2 IgG and $\operatorname{lgM}$ of solid $(n=14)$ and hematologic $(n=7)$ cancer patients and non-cancer patients ( $n=108)$. (c) (Left) Global UMAP projection of lymphocyte populations for all 45 patients pooled. (Right) Hierarchical clustering of Earth Mover's Distance (EMD) using Pearson correlation, calculated pairwise for lymphocyte populations. (d) UMAP projection of concatenated lymphocyte populations for each EMD cluster. (Yellow: High Density; Black; Low Density) (e) Heatmap showing expression patterns of various markers, stratified by EMD cluster. Heat scale calculated as column z-score of MFI. (f) Mortality, disease severity, and SARS-CoV-2 antibody data, stratified by EMD cluster (Cluster $5 \mathrm{n}=5$; Cluster 1,2,3,4 $\mathrm{n}=40$ ). Mortality significance determined by Pearson Chi Square test. Severity assessed with NIH ordinal scale for COVID-19 clinical severity (1: Death; 8: Normal Activity) ${ }^{15}$. (g) UMAP projections of concatenated lymphocyte populations for solid cancer, hematologic cancer, and non-cancer patients. (h) CD8 and CD4 T cell and B cell frequencies in healthy donors (HD) $(n=33)$, non-cancer $(n=108)$, solid cancer $(n=7)$, and heme cancer $(n=4)$. (i) UMAP projection of non-naive CD8 T cell clusters identified by FlowSOM. (j) (Top) UMAP projections of non-naïve CD8 T cells for non-cancer and cancer patients. (Bottom) UMAP projections indicating HLA-DR and CD38 protein expression on non-naive CD8 T cells for all patients pooled. (k) Frequency of activated FlowSOM clusters in HD ( $n=30)$, non-cancer $(n=110)$, and cancer patients $(n=8)$. (I) Representative flow plots and frequency of HLA-DR and CD38 co-expression in HD ( $n=30)$, non-cancer $(n=110)$, solid cancer $(n=7)$, and hematologic cancer $(n=3)$ patients. (All) Significance determined by Mann Whitney test: ${ }^{*} p<0.05,{ }^{* *} p<0.01,{ }^{* * *} p<0.001$, and ${ }^{* * * *} p<0.0001$. Median and $95 \% \mathrm{Cl}$ shown. 
Fig. 4
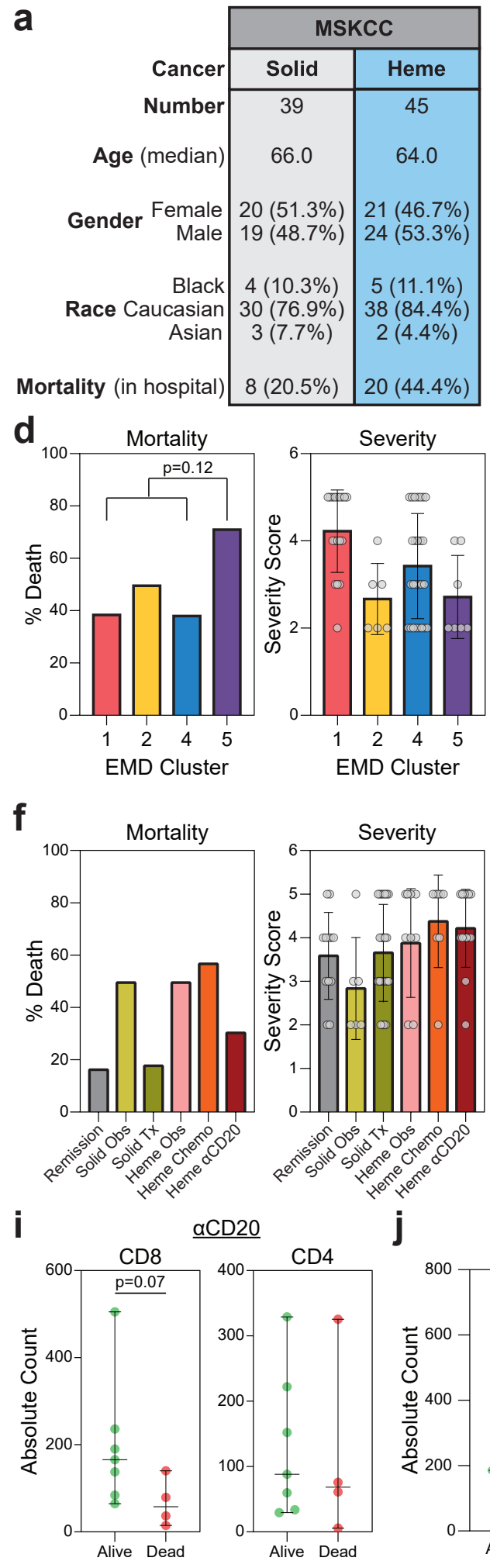
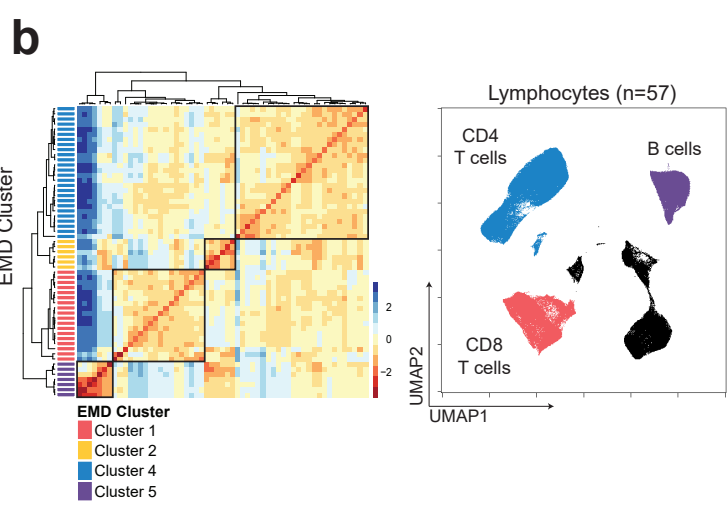

C
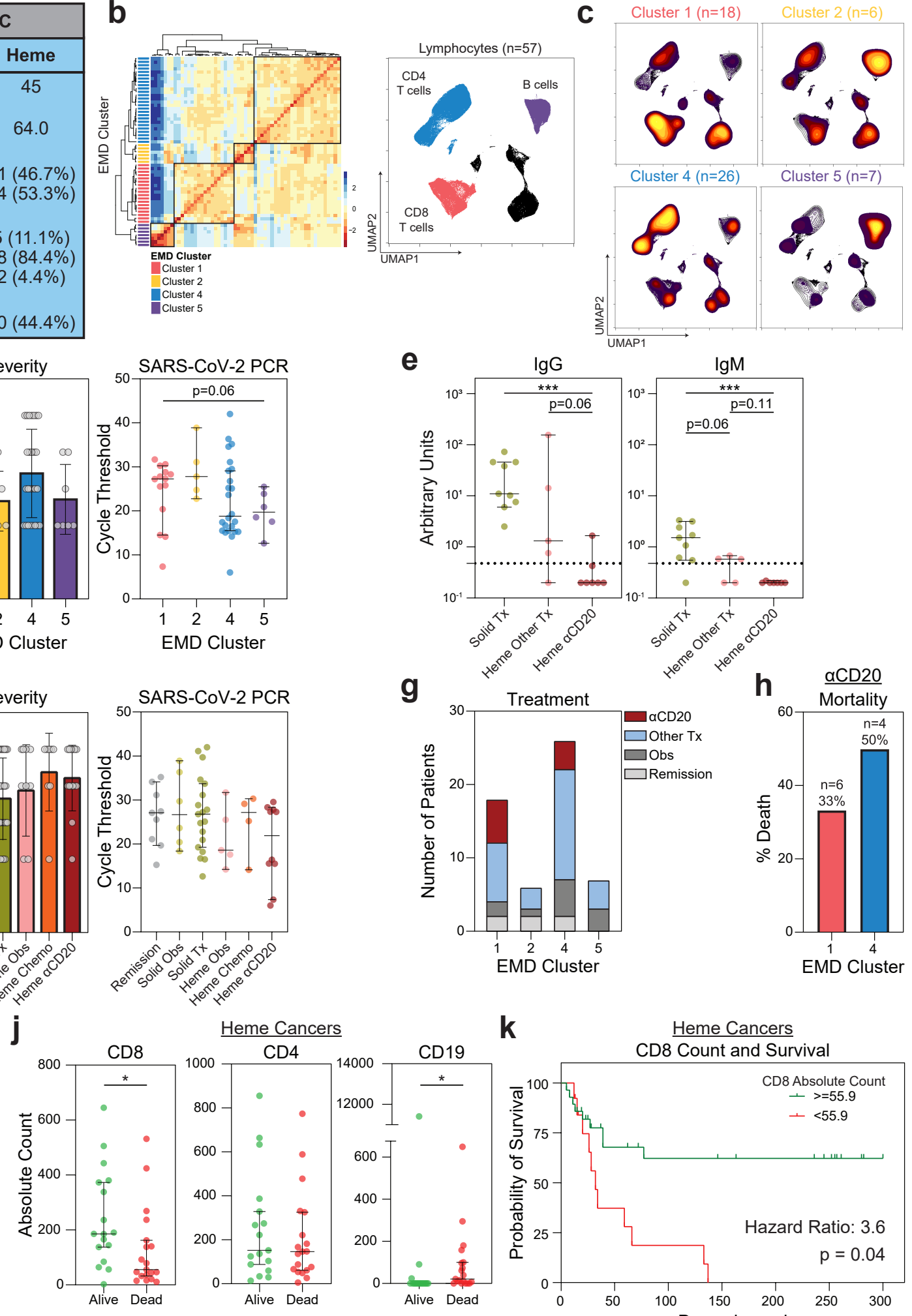

k
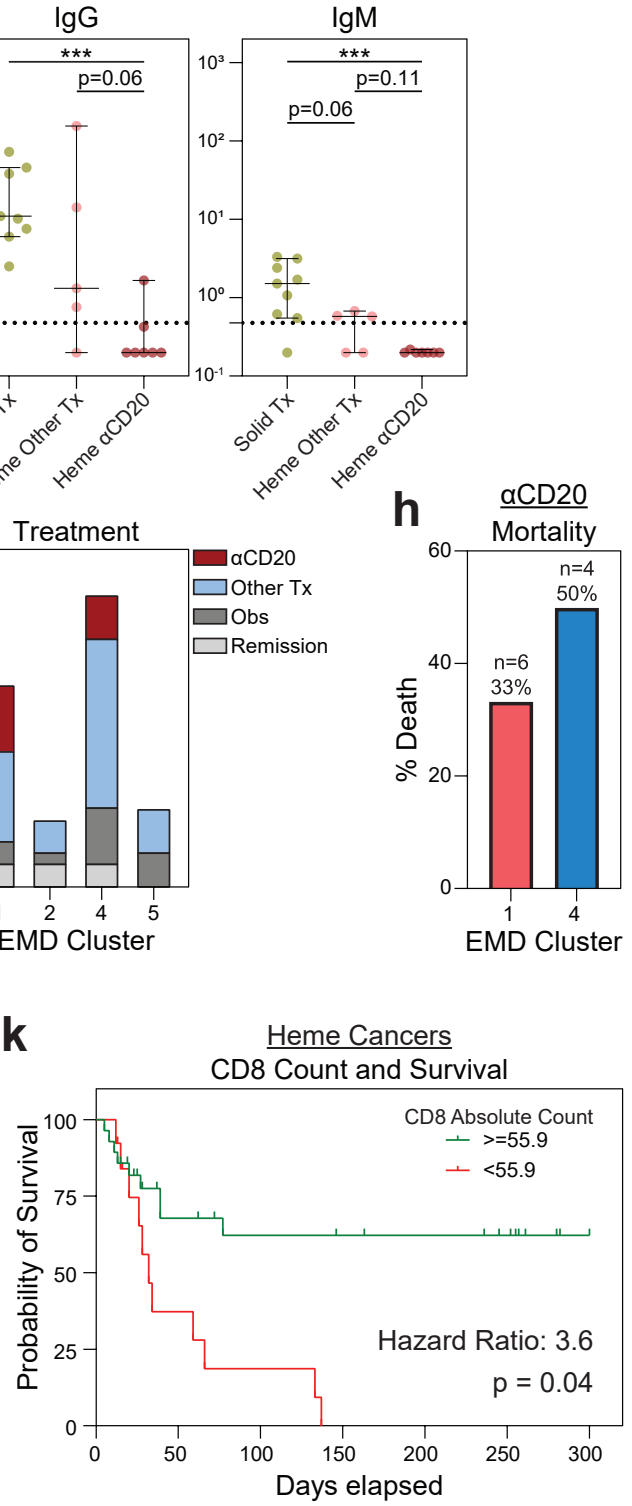

Fig. 4 | CD8 T cell counts associated with survival in hematologic cancer patients with COVID-19. (a) Demographic and mortality data of MSKCC cohort. (b) (Left) Hierarchical clustering of Earth Mover's Distance (EMD) using Pearson correlation, calculated pairwise for lymphocyte populations. (Right) Global UMAP projection of lymphocyte populations pooled. (c) UMAP projection of concatenated lymphocyte populations for each EMD cluster. (Yellow: High Density; Black: Low Density) (d) Mortality (Cluster $5 \mathrm{n=7}$; Cluster 1,2,4 n=50), severity, and RT-PCR cycle threshold (Cluster $1 n=14$; Cluster $2 n=5$; Cluster $4 n=24$; Cluster $5 n=6$ ) (Lower Ct: Higher viral load) stratified by EMD cluster. Mortality significance determined by Pearson Chi Square test. (e) Relative levels of SARS-CoV-2 IgG and IgM of patients with recent cancer treatments (solid tx n=9; heme $\alpha C D 20 n=7$; heme other $t x n=5$ ). (f) Mortality, severity, and RT-PCR cycle threshold stratified by cancer treatment (remission $n=9$; solid obs $n=6$; solid $t x n=19$; heme obs $n=5$; heme chemo $n=4$; heme $a C D 20 n=10$ ). Severity assessed with $N I H$ ordinal scale for COVID-19 clinical severity. (g) Recent cancer treatment of patients in each EMD cluster. (h) Mortality of patients treated with $\mathrm{B}$ cell depleting therapy in EMD cluster 1 (red) and EMD cluster 4 (blue). (i) Absolute CD8 and CD4 T cell counts in patients treated with B cell depleting therapy (alive $n=7$; dead $n=4$ ). (j) Absolute CD8 and CD4 T cell counts and B cell counts in hematologic cancer patients (alive $\mathrm{n}=17$; dead $\mathrm{n=18}$ ). (k) Kaplan-Meier curve for survival in hematologic cancer patients stratified by CD8 $\mathrm{T}$ cell counts (threshold = 55.9; log-rank hazard ratio) (>=55.9n=28; $<55.9 n=13)$. CD8 count threshold determined by Classification and Regression Tree (CART) analysis. (All) Significance determined by Mann Whitney test: ${ }^{*} p<0.05,{ }^{* *} p<0.01,{ }^{* * *} p<0.001$, and ${ }^{* * *} p<0.0001$. Median and $95 \% \mathrm{Cl}$ shown. 


\section{Extended Data Fig. 1}

a
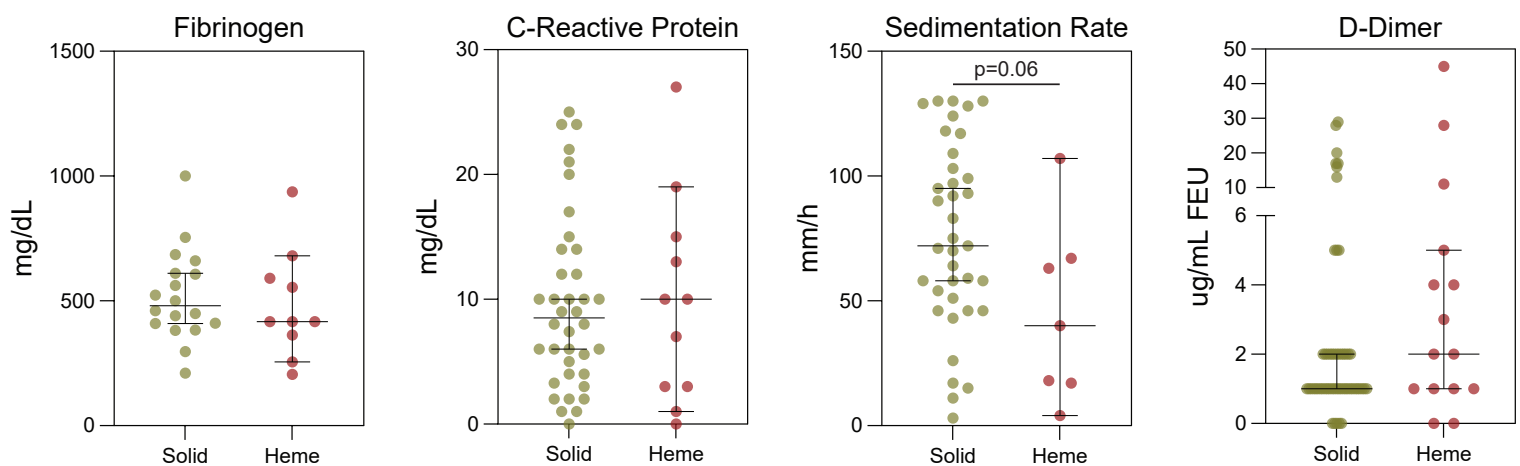

b
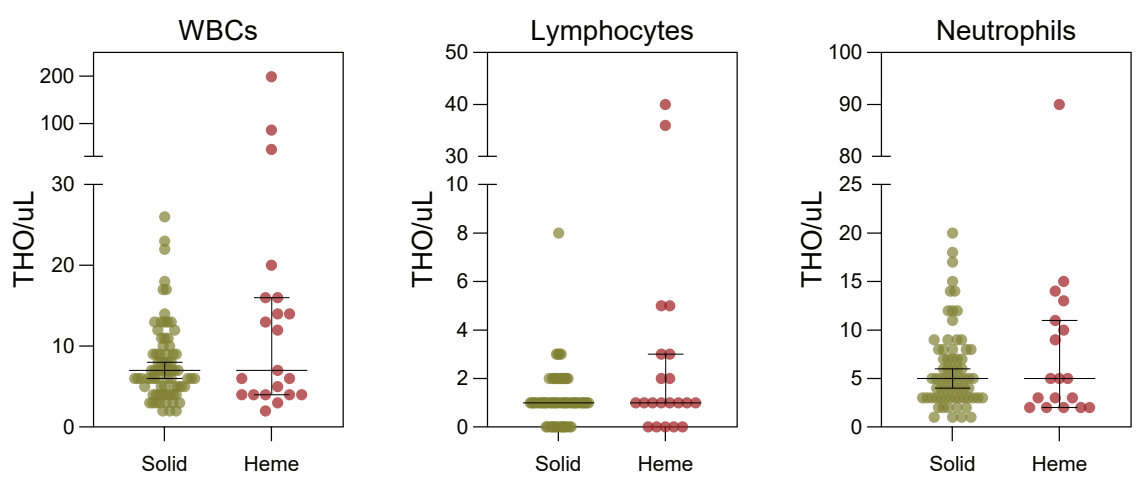

Extended Data Fig. 1 | Inflammatory markers and blood cell counts in cancer patients with COVID-19. Clinical laboratory values for (a) inflammatory markers and (b) cell counts in solid $(n=62)$ and hematologic $(n=21)$ cancer patients. (All) Significance determined by Mann Whitney test: ${ }^{*} p<0.05$, ${ }^{* *} p<0.01$, ${ }^{* * *} p<0.001$, and ${ }^{* * * *} p<0.0001$. Median and $95 \% \mathrm{Cl}$ shown. 


\section{Extended Data Fig. 2}
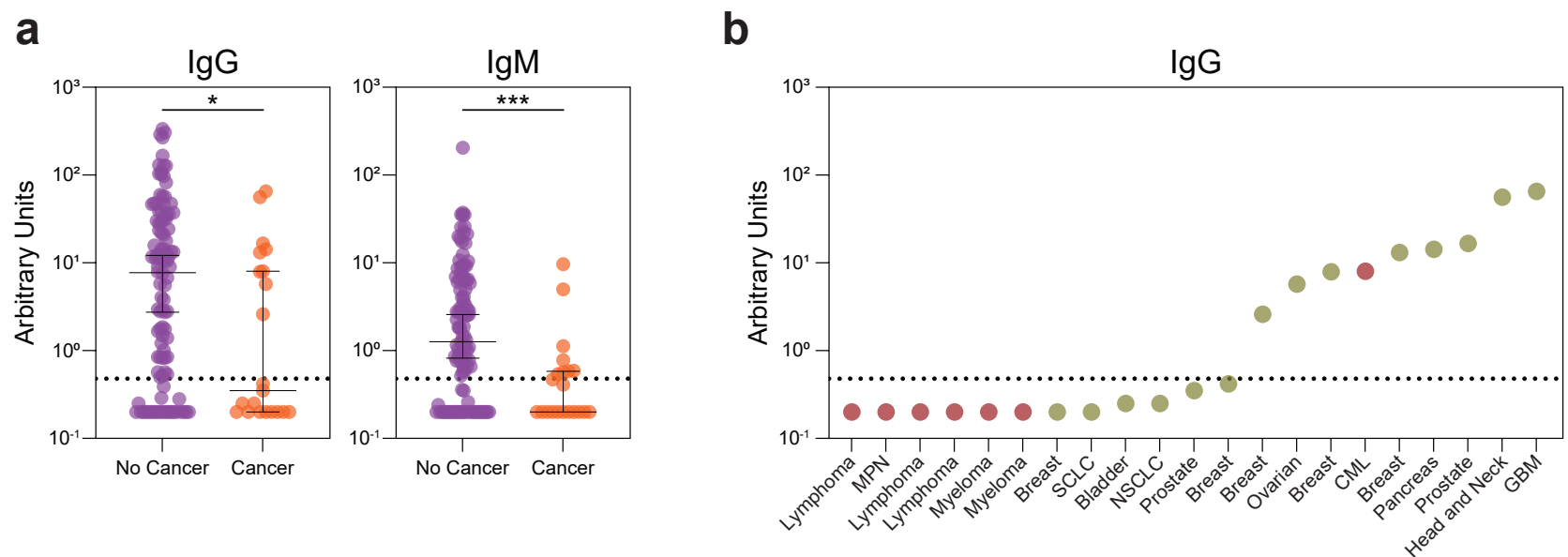

Extended Data Fig. 2 | SARS-CoV-2 antibody levels. (a) Relative levels of SARS-CoV-2 IgG and IgM in non-cancer $(n=108)$ and cancer $(n=21)$ patients. (b) Relative IgG levels in cancer patients. Each dot represents a cancer patient (Heme: Red; Solid: Yellow). (All) Significance determined by Mann Whitney test: ${ }^{*} p<0.05,{ }^{* *} p<0.01,{ }^{* * *} p<0.001$, and ${ }^{* * * *} p<0.0001$. Median and $95 \% \mathrm{Cl}$ shown. 


\section{Extended Data Fig. 3}

a
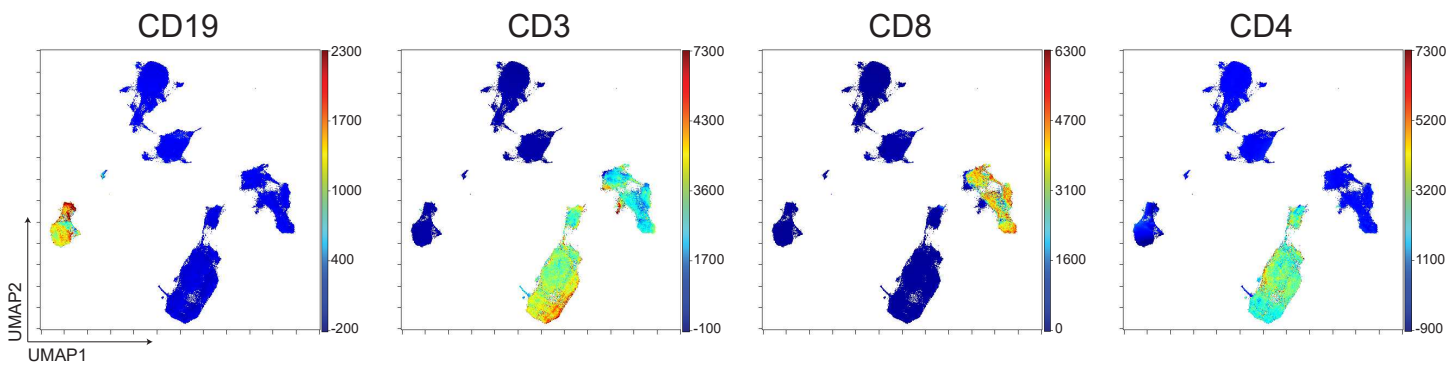

b
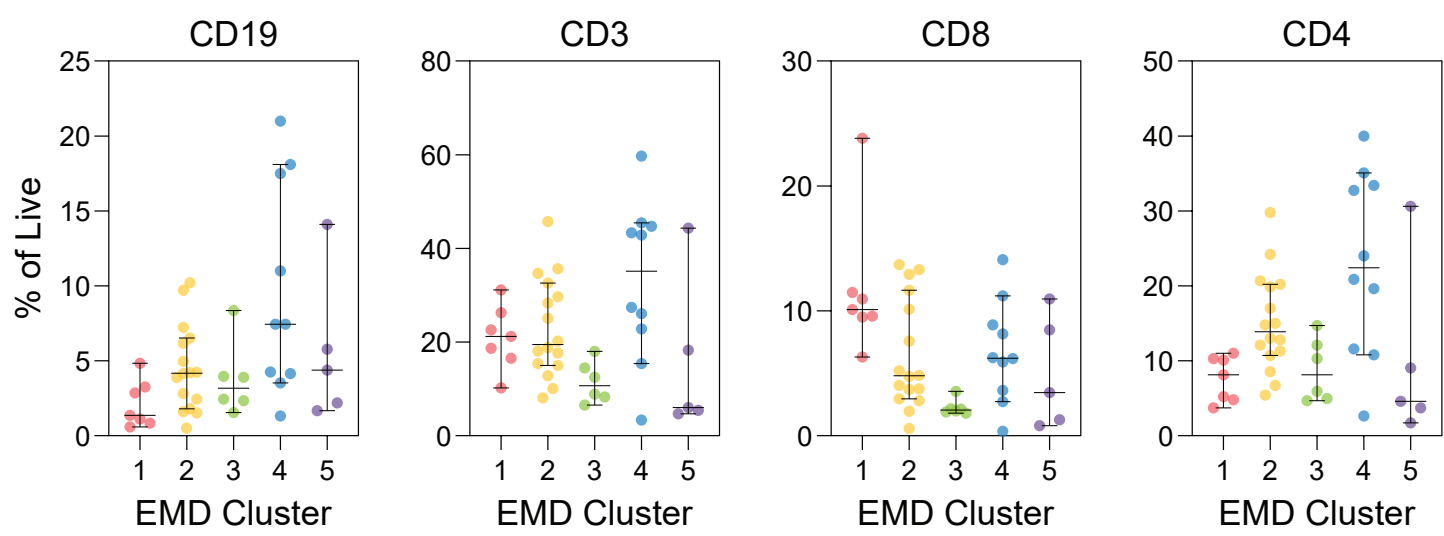

Extended Data Fig. 3 | Dimensionality reduction and EMD clustering of MESSI cohort. (a) UMAP projections of lymphocytes with indicated protein expression. (b) Frequencies of CD19+, CD3+, CD3+CD8+, and CD3+CD4+ cells of patients in each EMD cluster (Cluster $1 \mathrm{n}=7$; Cluster $2 \mathrm{n}=16$; Cluster $3 \mathrm{n}=6$; Cluster $4 \mathrm{n}=10$; Cluster 5 $\mathrm{n}=5$ ). (All) Median and $95 \% \mathrm{Cl}$ shown. 


\section{Extended Data Fig. 4}

a

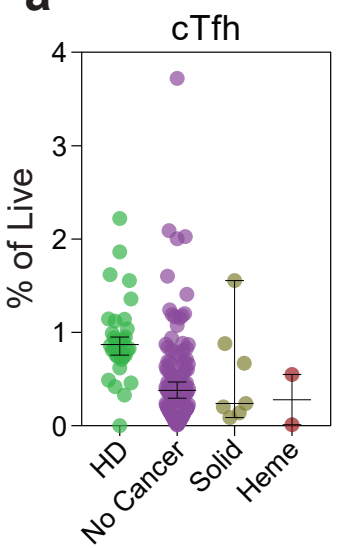

C

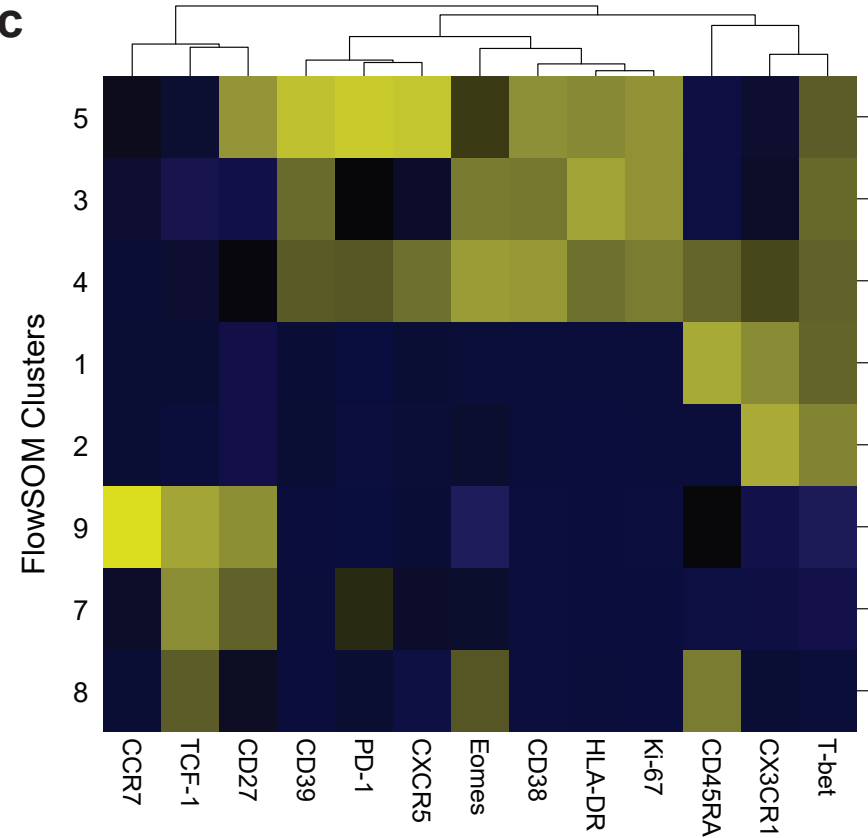

b
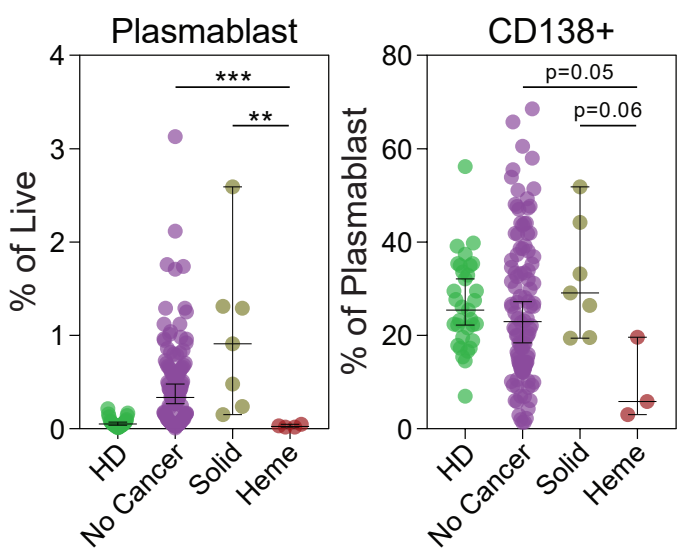
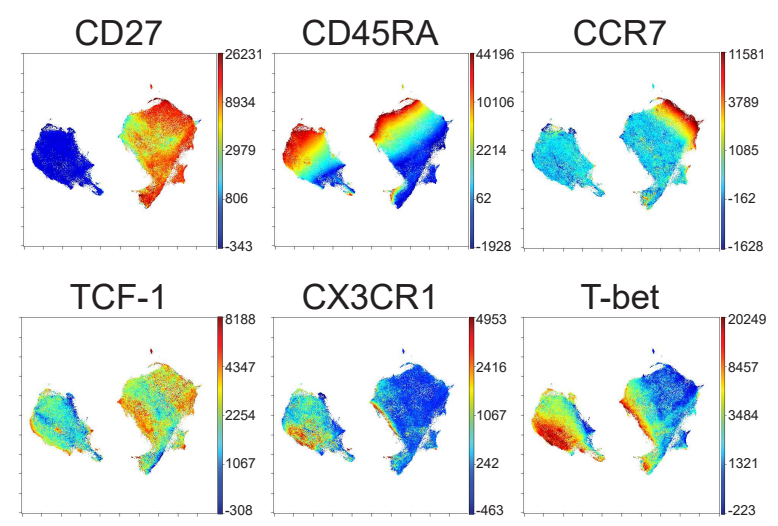

e
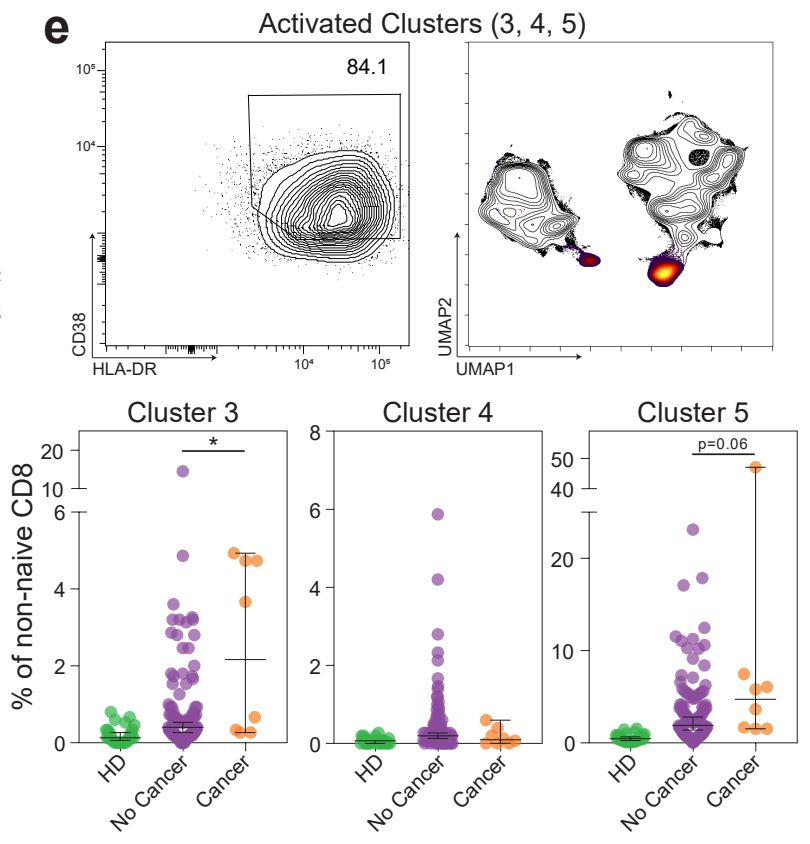

d
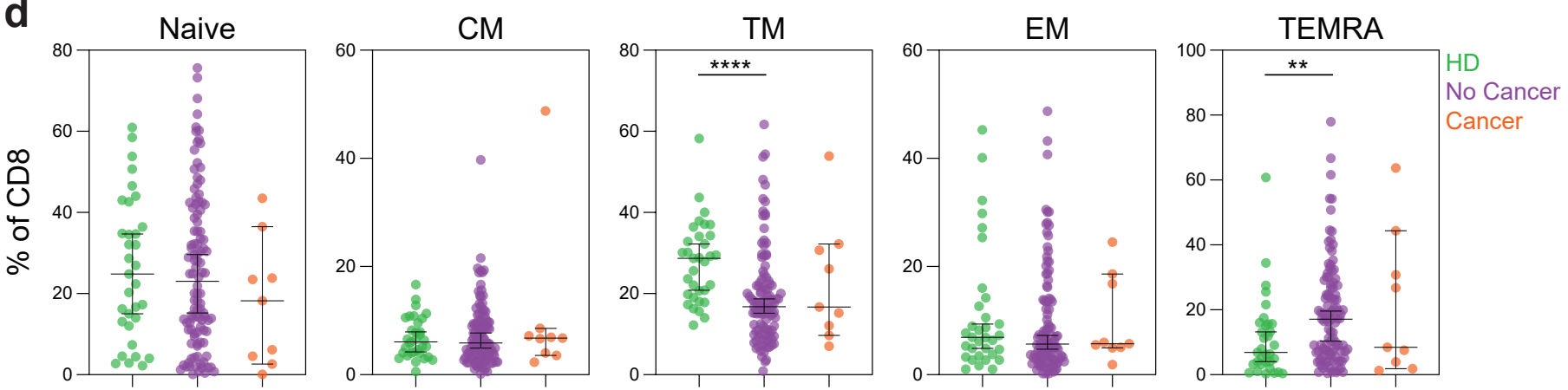

Extended Data Fig. 4 | Cellular phenotyping of COVID-19 patients with cancer. (a) Frequencies of circulating T follicular helper cells (cTfh), plasmablasts, and CD138 expression on plasmablasts (HD n=33; non-cancer $n=108$; solid cancer $n=7$; heme cancer $n=3$ ). (b) UMAP projection of non-naïve CD8 T cells with indicated protein expression. (c) Heatmap showing expression patterns of various markers, stratified by FlowSOM clusters. Heat scale calculated as column z-score of MFI. (d) Frequencies of CD8 subsets: naive (CD45RA+CD27+CCR7+), central memory (CD45RA-CD27+CCR7+), transition memory (CD45RA-CD27+CCR7-), effector memory (CD45RA-CD27-CCR7-), and TEMRA (CD45RA+CD27-CCR7-) (HD n=33; non-cancer $n=108$; cancer $n=9)$. (e) (Top) HLA-DR and CD38 coexpression in concatenated activated clusters (3, 4, and 5 ) and associated UMAP localization. (Bottom) Frequency of activated clusters (3, 4, and 5 ) in each patient (HD n=30; non-cancer $n=110$; solid-cancer $n=8$ ). (All) Significance determined by Mann Whitney test: ${ }^{*} p<0.05,{ }^{* *} p<0.01,{ }^{* * *} p<0.001$, and ${ }^{* * * *} p<0.0001$. Median and $95 \% \mathrm{Cl}$ shown. 


\section{Extended Data Fig. 5}
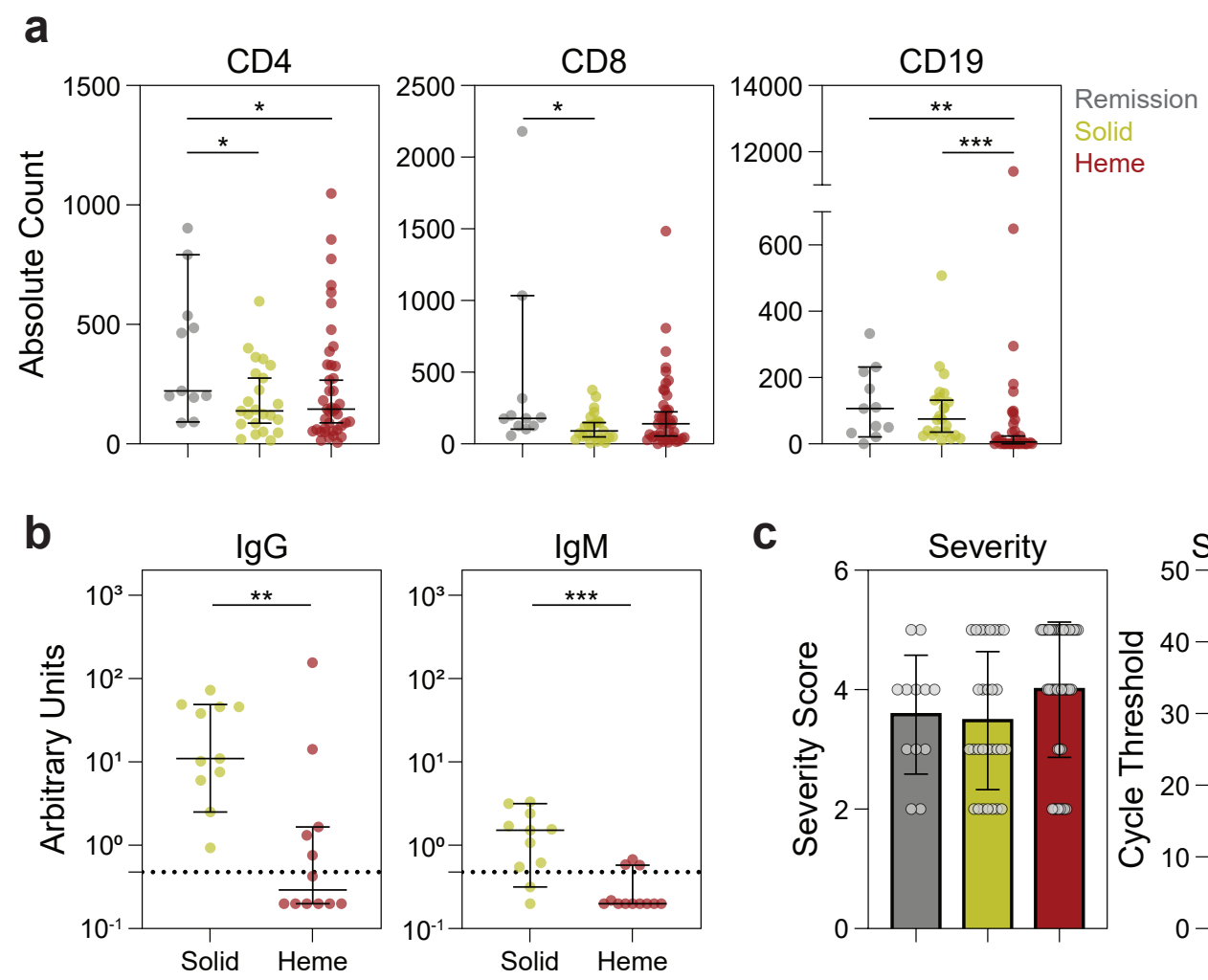

C
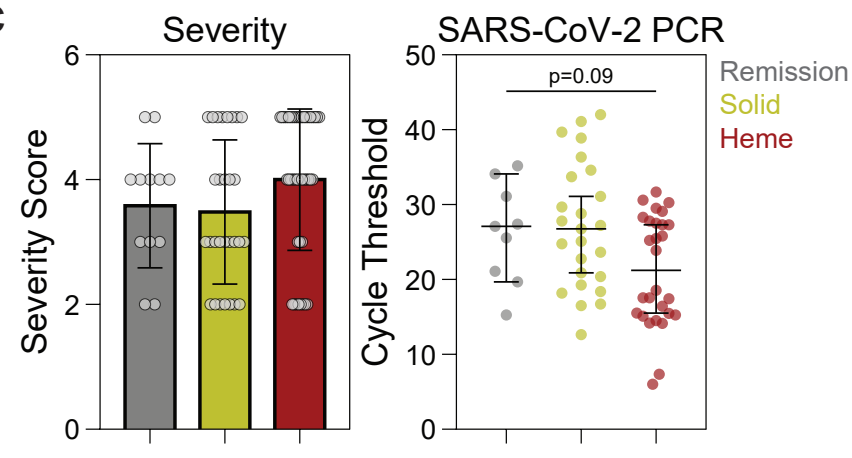

d

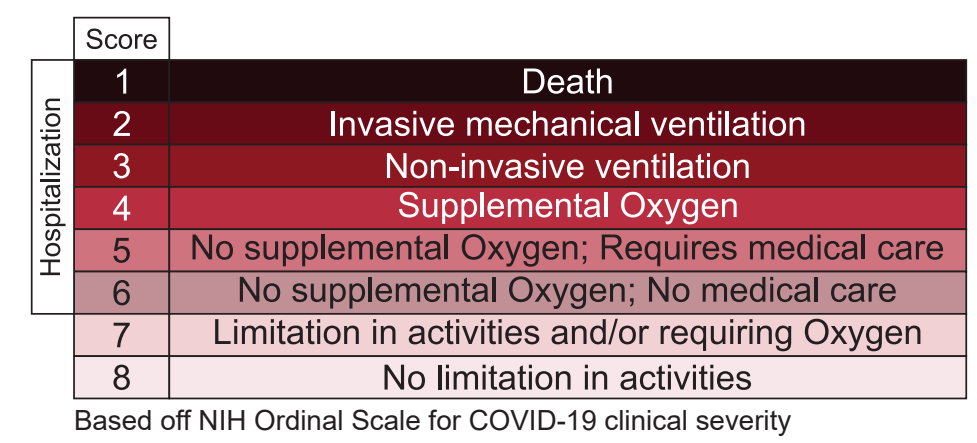

Extended Data Fig. 5 | Cellular, serologic, and clinical features in solid and hematologic cancer patients with COVID-19. (a) Absolute counts of CD4, CD8, and CD19 expression in remission $(n=11)$, solid cancer $(n=23)$, and hematologic cancer $(n=41)$ patients. (b) Relative levels of SARS-CoV-2 IgG and IgM in solid $(n=11)$ and hematologic cancer $(n=14)$ patients. (c) Severity ( $\mathrm{NIH}$ ordinal scale for COVID-19 clinical severity) and RT-PCR cycle threshold (remission $n=9$; solid $n=25$; heme $n=28$ ) (Lower Ct: Higher viral load). (d) NIH ordinal scale for COVID-19 clinical severity. (All) Significance determined by Mann Whitney test: ${ }^{*} p<0.05,{ }^{* *} p<0.01,{ }^{* * *} p<0.001$, and ${ }^{* * * *} p<0.0001$. Median and 95\% Cl shown. 


\section{Extended Data Fig. 6}

a
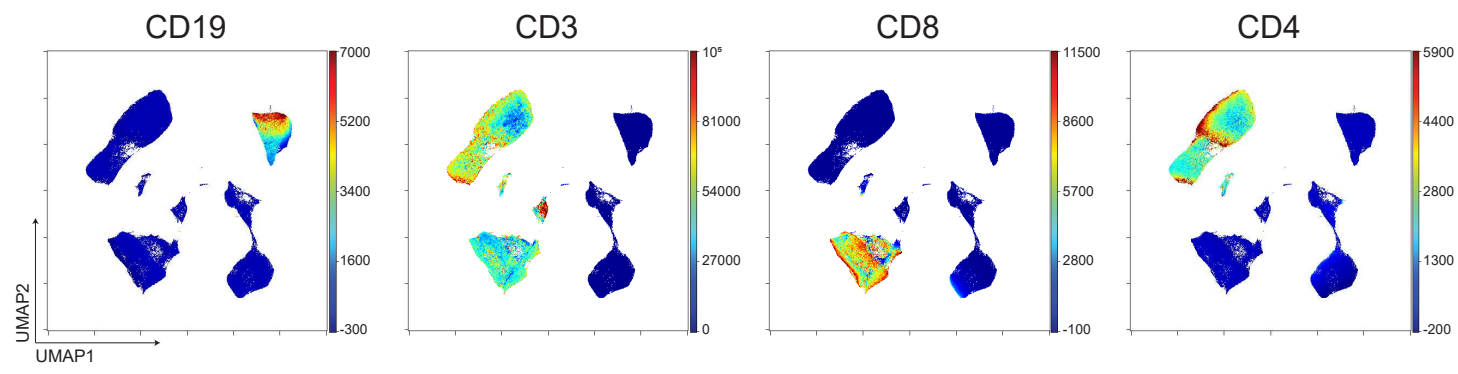

b
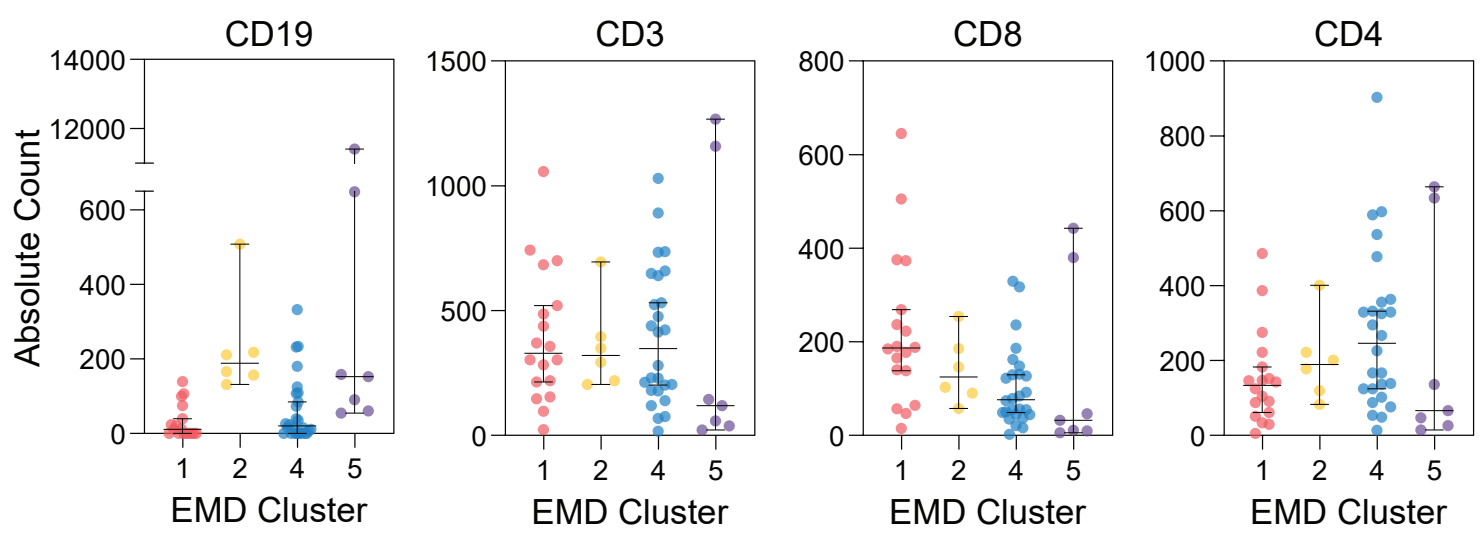

Extended Data Fig. 6 | Dimensionality reduction and EMD clustering of MSKCC cohort. (a) UMAP projections of lymphocytes with indicated protein expression. (b) Absolute counts of CD19+, CD3+, CD3+CD8+, and CD3+CD4+ cells of patients in each EMD cluster (Cluster $1 \mathrm{n}=18$; Cluster $2 \mathrm{n}=6$; Cluster $3 \mathrm{n}=26$; Cluster $4 \mathrm{n=7}$ ). (All) Median and $95 \% \mathrm{Cl}$ shown. 


\section{Extended Data Fig. 7}

a

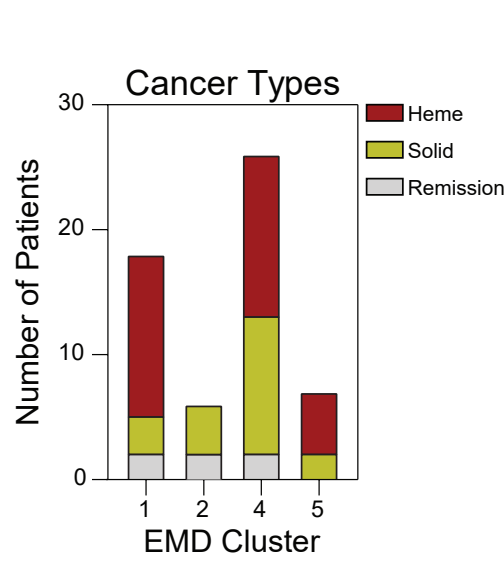

EMD Cluster 4

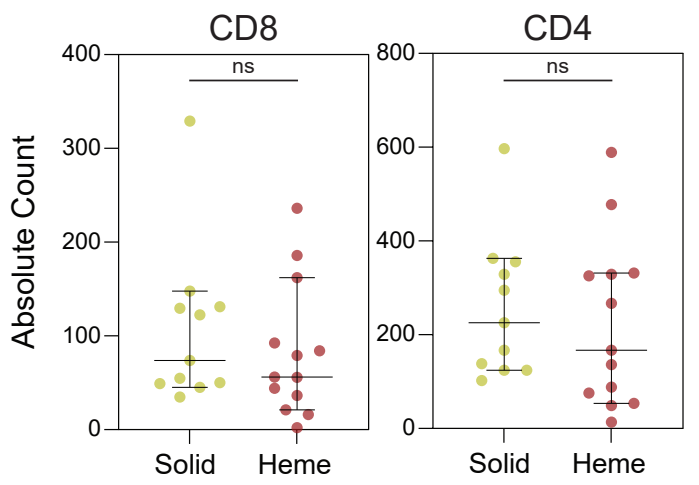

$\underline{\text { Heme Cancers }}$

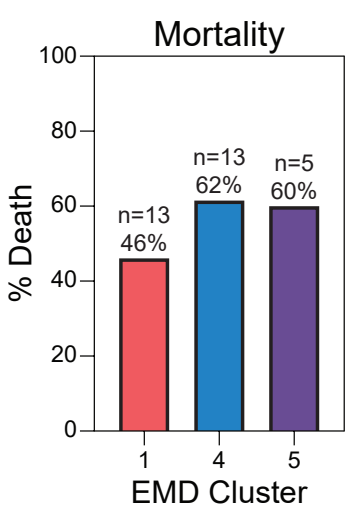

d

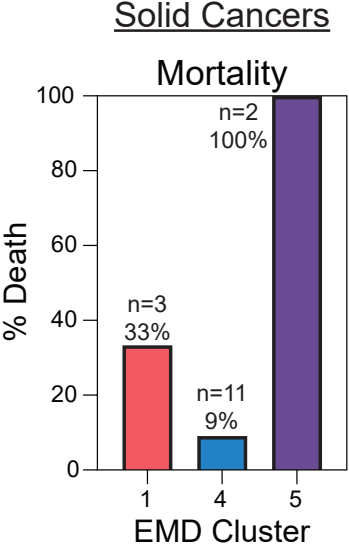

b EMD Cluster 4

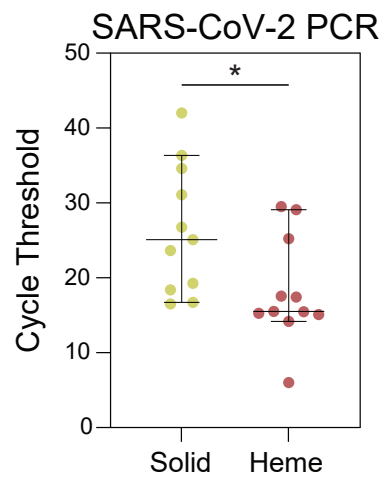

C

Extended Data Fig. 7 | EMD Cluster 4 drives differences in mortality between hematologic and solid cancer patients. (a) (Left) Number of patients with hematologic, solid, and remission cancer statuses within each EMD cluster. (Right) Mortality of patients within each EMD cluster for hematologic and solid cancers. (b) RT-PCR cycle threshold of solid and heme cancer patients in EMD cluster 4 (solid $n=11$; heme $n=11$ ). (c) Absolute CD8 and CD4 T cell counts for subjects in EMD cluster 4 stratified by solid $(n=11)$ and heme $(n=13)$ cancer. (d) Global UMAP projections of lymphocytes for subjects in EMD cluster 4: (Left) Hematologic cancer; (Middle) Solid cancer. (Right) Absolute B cell counts for subjects in EMD cluster 4 stratified by solid $(n=11)$ and heme $(n=13)$ cancer. (All) Significance determined by Mann Whitney test: ${ }^{*} p<0.05,{ }^{* *} p<0.01$, ${ }^{* * *} p<0.001$, and ${ }^{* * *} p<0.0001$. Median and $95 \% \mathrm{Cl}$ shown. 


\section{Extended Data Fig. 8}

a
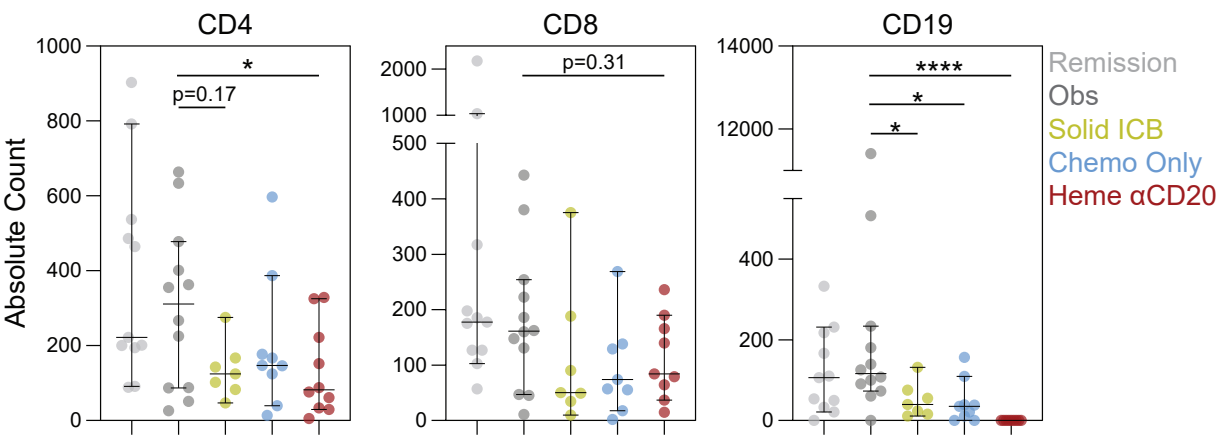

b
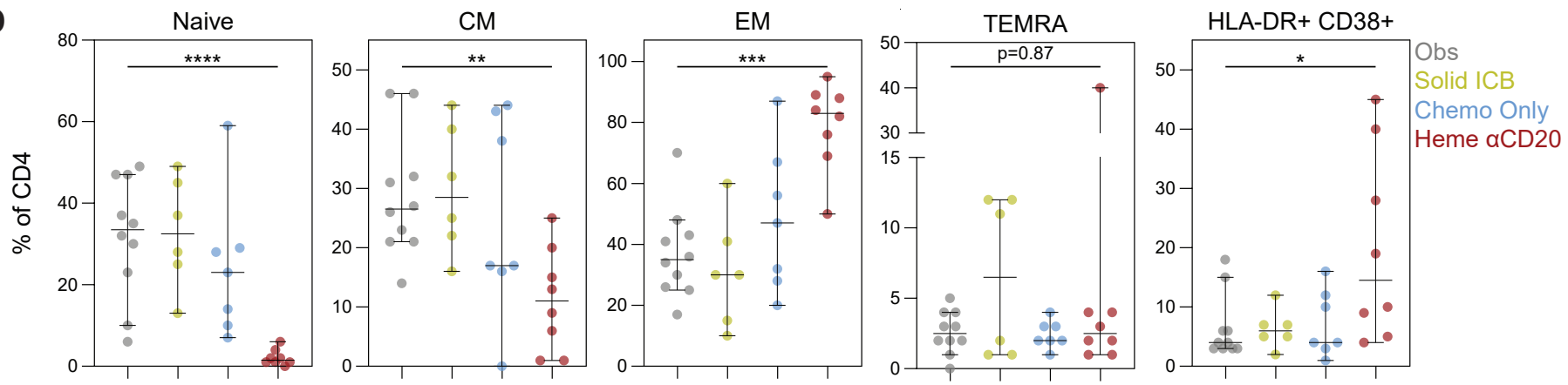

C
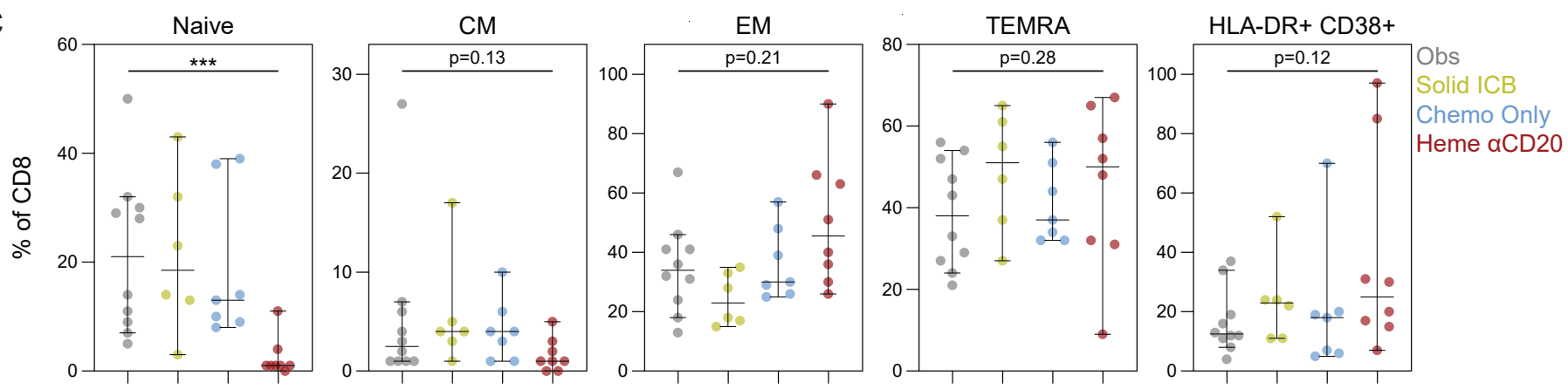

Extended Data Fig. 8 | Effect of cancer treatment on T cell differentiation in cancer patients with COVID-19. (a) Absolute counts of CD4, CD8, and CD19 expressing cells. Frequencies of (b) CD4 and (c) CD8 T cell subsets in cancer patients treated with immune checkpoint blockade therapies, chemotherapies, and $B$ cell depleting therapies. Naive (CD45RA+CCR7+), CM (CD45RA-CCR7+), EM (CD45RA-CCR7-), TEMRA (CD45RA+CCR7-). (All) Remission $n=11$, obs $n=12$, chemo only $n=9$, solid ICB $n=7$, and heme $\alpha C D 20$ $\mathrm{n}=10$. Significance determined by Mann Whitney test: ${ }^{*} \mathrm{p}<0.05,{ }^{* *} \mathrm{p}<0.01,{ }^{* * *} \mathrm{p}<0.001$, and ${ }^{* * * *} \mathrm{p}<0.0001$. Median and $95 \% \mathrm{Cl}$ shown. 


\section{Extended Data Fig. 9}

a

$\underline{\alpha C D 20}$

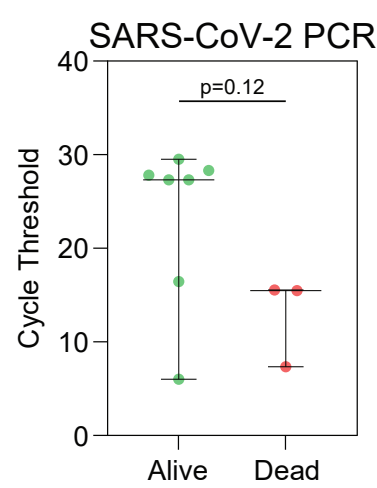

b

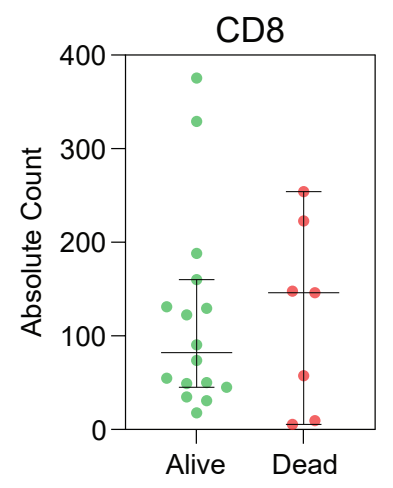

Solid Cancers

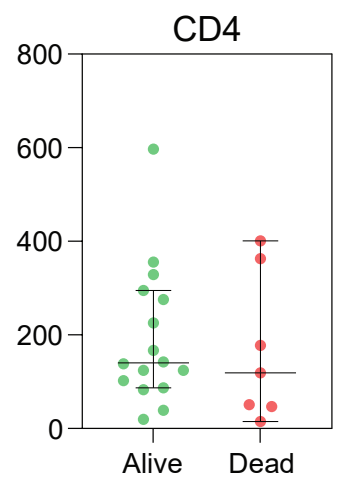

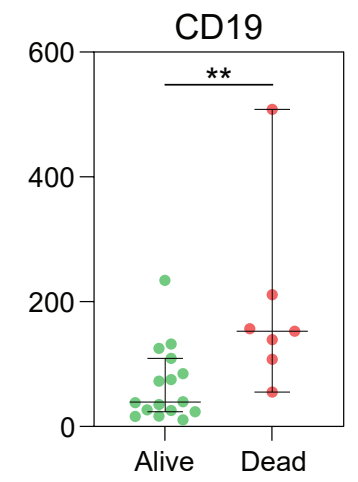

Extended Data Fig. 9 | Association of mortality with cell counts and viral load. (a) RT-PCR cycle threshold of patients treated with aCD20 therapy (alive $n=7$; dead $n=3$ ). (b) Absolute counts of CD8+, CD4+, and CD19+ cells in solid cancer patients (alive $n=16$; dead $n=7$ ). (All) Significance determined by Mann Whitney test: ${ }^{*} p<0.05,{ }^{* *} p<0.01,{ }^{* * *} p<0.001$, and ${ }^{* * * *} p<0.0001$. Median and $95 \% \mathrm{Cl}$ shown. 
Supplementary Table 1 | COPE: Patient demographics and clinical characteristics by tumor type.

\begin{tabular}{|c|c|c|}
\hline & $\begin{array}{c}\text { Solid } \\
(\mathrm{N}=78)\end{array}$ & $\begin{array}{l}\text { Heme } \\
(\mathrm{N}=22)\end{array}$ \\
\hline Age, median (IQR) & $70.5(57-78)$ & $64.5(60-77)$ \\
\hline Gender, female & $39(50.0 \%)$ & $9(40.9 \%)$ \\
\hline \multicolumn{3}{|l|}{ Race } \\
\hline White & $24(30.8 \%)$ & $9(40.9 \%)$ \\
\hline Non-white & $49(62.8 \%)$ & $12(54.6 \%)$ \\
\hline Unknown & $5(6.41 \%)$ & $1(4.55 \%)$ \\
\hline Smoking History, Current/Prior & $44(56.4 \%)$ & $13(59.1 \%)$ \\
\hline \multicolumn{3}{|l|}{ Comorbidities } \\
\hline Cardiac & $63(80.8 \%)$ & $15(68.2 \%)$ \\
\hline Pulmonary & $38(48.7 \%)$ & $3(13.6 \%)$ \\
\hline Use of immunosuppressive drugs ${ }^{+}$ & $23(29.5 \%)$ & $7(31.8 \%)$ \\
\hline BMI, median (IQR) & $26.6(23.2-30.9)$ & $28.7(24.0-33.4)$ \\
\hline Cancer Status, Active $^{++}$ & $32(41.0 \%)$ & $14(63.6 \%)$ \\
\hline \multicolumn{3}{|l|}{ Cancer Treatment } \\
\hline \multicolumn{3}{|l|}{ Treatment in last 3 months } \\
\hline Cytotoxic Chemotherapy & $16(20.5 \%)$ & $8(36.4 \%)$ \\
\hline Hormone therapy & $15(19.2 \%)$ & $0(0.00 \%)$ \\
\hline Active Surveillance/surgery & $43(55.1 \%)$ & $10(45.5 \%)$ \\
\hline Other* & $4(5.13 \%)$ & $4(18.2 \%)$ \\
\hline ECOG Performance Status & $\mathrm{N}=58$ & $\mathrm{~N}=15$ \\
\hline $0-1$ & $28(48.3 \%)$ & $9(60.0 \%)$ \\
\hline 2 & $9(15.5 \%)$ & $4(26.7 \%)$ \\
\hline $3-4$ & $21(36.2 \%)$ & $2(13.3 \%)$ \\
\hline $\begin{array}{l}{ }^{*} \text { Single agent immunotherapy, targeted thera } \\
{ }^{+} \text {Exposure to immunosuppressive medication } \\
{ }^{+} \text {Diagnosis or treatment within } 6 \text { months }\end{array}$ & & \\
\hline
\end{tabular}


Supplementary Table 2 | MESSI: Patient demographics and clinical characteristics by cancer status.

\begin{tabular}{|c|c|c|c|}
\hline & $\begin{array}{c}\text { Non-Cancer } \\
(\mathbf{N}=108)\end{array}$ & $\begin{array}{l}\text { Cancer } \\
(\mathrm{N}=22)\end{array}$ & $\begin{array}{l}\text { Overall } \\
(\mathrm{N}=130)\end{array}$ \\
\hline \multicolumn{4}{|l|}{ Gender } \\
\hline Female & $49(45.4 \%)$ & $14(63.6 \%)$ & $63(48.5 \%)$ \\
\hline Male & $59(54.6 \%)$ & $8(36.4 \%)$ & $67(51.5 \%)$ \\
\hline Age (median) & 60 & 66 & 60.5 \\
\hline \multicolumn{4}{|l|}{ Race } \\
\hline Asian & $5(4.6 \%)$ & $1(4.5 \%)$ & $6(4.6 \%)$ \\
\hline Black & $74(68.5 \%)$ & $16(72.7 \%)$ & $90(69.2 \%)$ \\
\hline White & $28(25.9 \%)$ & $5(22.7 \%)$ & $33(25.4 \%)$ \\
\hline Pacific Islander & $1(0.9 \%)$ & $0(0 \%)$ & $1(0.8 \%)$ \\
\hline Symptoms (Days before hospitalization) (median) & 9 & 8 & 9 \\
\hline Severity (At hospitalization) (median) & 3.5 & 3 & 3 \\
\hline Mortality (28 days) & $12(11.1 \%)$ & $8(36.4 \%)$ & $20(15.4 \%)$ \\
\hline
\end{tabular}


Supplementary Table 3 | MESSI: Patient demographics and clinical characteristics by cancer type.

\begin{tabular}{|c|c|c|c|}
\hline & $\begin{array}{l}\text { Heme } \\
(\mathrm{N}=7)\end{array}$ & $\begin{array}{l}\text { Solid } \\
(\mathrm{N}=15)\end{array}$ & $\begin{array}{l}\text { Overall } \\
(\mathrm{N}=22)\end{array}$ \\
\hline \multicolumn{4}{|l|}{ Gender } \\
\hline Female & $4(57.1 \%)$ & $10(66.7 \%)$ & $14(63.6 \%)$ \\
\hline Male & $3(42.9 \%)$ & $5(33.3 \%)$ & $8(36.4 \%)$ \\
\hline Age (median) & 67 & 65 & 66 \\
\hline \multicolumn{4}{|l|}{ Race } \\
\hline Asian & $0(0 \%)$ & $1(6.7 \%)$ & $1(4.5 \%)$ \\
\hline Black & $5(71.4 \%)$ & $11(73.3 \%)$ & $16(72.7 \%)$ \\
\hline White & $2(28.6 \%)$ & $3(20.0 \%)$ & $5(22.7 \%)$ \\
\hline Symptoms (Days before hospitalization) (median) & 9 & 7.5 & 8 \\
\hline Severity (At hospitalization) (median) & 3 & 4 & 3 \\
\hline Mortality (28 days) & $2(28.6 \%)$ & $6(40.0 \%)$ & $8(36.4 \%)$ \\
\hline \multicolumn{4}{|l|}{ COVID Treatments } \\
\hline Remdesivir & $1(14.3 \%)$ & $2(13.3 \%)$ & $3(13.6 \%)$ \\
\hline Convalescent Plasma & $1(14.3 \%)$ & $3(20.0 \%)$ & $4(18.2 \%)$ \\
\hline Early Steroids & $4(57.1 \%)$ & $5(33.3 \%)$ & $9(40.9 \%)$ \\
\hline
\end{tabular}


Supplementary Table 4 | MESSI: Cancer type and cancer treatment.

\begin{tabular}{|l|c|c|c|}
\hline \multicolumn{2}{|c|}{$\begin{array}{c}\text { Heme } \\
\text { (N=7) }\end{array}$} & & Solid \\
\hline Cancer Type & $1(14.3 \%)$ & $0(0 \%)$ & $1(4.5 \%)$ \\
\hline CML & $1(14.3 \%)$ & $0(0 \%)$ & $1(4.5 \%)$ \\
\hline CTCL & $1(14.3 \%)$ & $0(0 \%)$ & $1(4.5 \%)$ \\
\hline Lymphoma & $1(14.3 \%)$ & $0(0 \%)$ & $1(4.5 \%)$ \\
\hline Mantle Cell Lymphoma & $1(14.3 \%)$ & $0(0 \%)$ & $1(4.5 \%)$ \\
\hline MM & $1(14.3 \%)$ & $0(0 \%)$ & $1(4.5 \%)$ \\
\hline MPN & $1(14.3 \%)$ & $0(0 \%)$ & $1(4.5 \%)$ \\
\hline Myeloma & $0(0 \%)$ & $1(6.7 \%)$ & $1(4.5 \%)$ \\
\hline Bladder & $0(0 \%)$ & $6(40.0 \%)$ & $6(27.3 \%)$ \\
\hline Breast & $0(0 \%)$ & $1(6.7 \%)$ & $1(4.5 \%)$ \\
\hline GBM & $0(0 \%)$ & $1(6.7 \%)$ & $1(4.5 \%)$ \\
\hline Head and Neck & $0(0 \%)$ & $1(6.7 \%)$ & $1(4.5 \%)$ \\
\hline NSCLC & $0(0 \%)$ & $1(6.7 \%)$ & $1(4.5 \%)$ \\
\hline Ovarian & $0(0 \%)$ & $1(6.7 \%)$ & $1(4.5 \%)$ \\
\hline Pancreas & $0(0 \%)$ & $2(13.3 \%)$ & $2(9.1 \%)$ \\
\hline Prostate & $0(0 \%)$ & $1(6.7 \%)$ & $1(4.5 \%)$ \\
\hline SCLC & & & \\
\hline Cancer Treatment & $1(14.3 \%)$ & $0(0 \%)$ & $1(4.5 \%)$ \\
\hline aCD20 + Chemotherapy & $5(71.4 \%)$ & $4(26.7 \%)$ & $9(40.9 \%)$ \\
\hline Chemo & $1(14.3 \%)$ & $1(6.7 \%)$ & $2(9.1 \%)$ \\
\hline None & $0(0 \%)$ & $1(6.7 \%)$ & $1(4.5 \%)$ \\
\hline Chemotherapy + Radiation & $0(0 \%)$ & $4(26.7 \%)$ & $4(18.2 \%)$ \\
\hline Hormonal & $0(0 \%)$ & $1(6.7 \%)$ & $1(4.5 \%)$ \\
\hline Hormonal + CDK Inhibitor & $0(0 \%)$ & $1(6.7 \%)$ & $1(4.5 \%)$ \\
\hline Hormonal + Radiation & $0(0 \%)$ & $3(20.0 \%)$ & $3(13.6 \%)$ \\
\hline ICB & & & \\
\hline
\end{tabular}


Supplementary Table 5 | MSKCC: Patient demographics and clinical characteristics by cancer type.

\begin{tabular}{|c|c|c|c|}
\hline & $\begin{array}{l}\text { Heme } \\
(\mathrm{N}=45)\end{array}$ & $\begin{array}{c}\text { Solid } \\
(N=39)\end{array}$ & $\begin{array}{l}\text { Overall } \\
(\mathrm{N}=84)\end{array}$ \\
\hline \multicolumn{4}{|l|}{ Gender } \\
\hline Female & $21(46.7 \%)$ & $20(51.3 \%)$ & $41(48.8 \%)$ \\
\hline Male & $24(53.3 \%)$ & $19(48.7 \%)$ & $43(51.2 \%)$ \\
\hline Age (median) & 64 & 66 & 65 \\
\hline \multicolumn{4}{|l|}{ Race } \\
\hline Asian & $2(4.4 \%)$ & $3(7.7 \%)$ & $5(6.0 \%)$ \\
\hline Black & $5(11.1 \%)$ & $4(10.3 \%)$ & $9(10.7 \%)$ \\
\hline White & $38(84.4 \%)$ & $30(76.9 \%)$ & $68(81.0 \%)$ \\
\hline Disease Severity (median) & 4 & 3 & 4 \\
\hline Mortality (In hospital) & $20(44.4 \%)$ & $8(20.5 \%)$ & $28(33.3 \%)$ \\
\hline \multicolumn{4}{|l|}{ COVID Treatments } \\
\hline Remdesivir & $12(26.7 \%)$ & $6(15.4 \%)$ & $18(21.4 \%)$ \\
\hline Convalescent Plasma & $25(55.6 \%)$ & $14(35.9 \%)$ & $39(46.4 \%)$ \\
\hline Early Steroids & $17(37.8 \%)$ & $21(53.8 \%)$ & $38(45.2 \%)$ \\
\hline
\end{tabular}


Supplementary Table 6 | MSKCC: Cancer type and cancer treatment.

\begin{tabular}{|c|c|c|c|}
\hline & $\begin{array}{l}\text { Heme } \\
(\mathrm{N}=45)\end{array}$ & $\begin{array}{l}\text { Solid } \\
(\mathrm{N}=39)\end{array}$ & $\begin{array}{l}\text { Overall } \\
(\mathrm{N}=84)\end{array}$ \\
\hline \multicolumn{4}{|l|}{ Cancer Type } \\
\hline ALL & $4(8.9 \%)$ & $0(0 \%)$ & $4(4.8 \%)$ \\
\hline AML & $6(13.3 \%)$ & $0(0 \%)$ & $6(7.1 \%)$ \\
\hline CLL & $4(8.9 \%)$ & $0(0 \%)$ & $4(4.8 \%)$ \\
\hline Lymphoma & $23(51.1 \%)$ & $0(0 \%)$ & $23(27.4 \%)$ \\
\hline MDS/Myelofibrosis & $3(6.7 \%)$ & $0(0 \%)$ & $3(3.6 \%)$ \\
\hline Myeloma & $5(11.1 \%)$ & $0(0 \%)$ & $5(6.0 \%)$ \\
\hline Bladder & $0(0 \%)$ & $2(5.1 \%)$ & $2(2.4 \%)$ \\
\hline Breast & $0(0 \%)$ & $8(20.5 \%)$ & $8(9.5 \%)$ \\
\hline CNS & $0(0 \%)$ & $3(7.7 \%)$ & $3(3.6 \%)$ \\
\hline Colorectal & $0(0 \%)$ & $5(12.8 \%)$ & $5(6.0 \%)$ \\
\hline GYN & $0(0 \%)$ & $3(7.7 \%)$ & $3(3.6 \%)$ \\
\hline Head and Neck & $0(0 \%)$ & $1(2.6 \%)$ & $1(1.2 \%)$ \\
\hline Kidney & $0(0 \%)$ & $1(2.6 \%)$ & $1(1.2 \%)$ \\
\hline Liver & $0(0 \%)$ & $1(2.6 \%)$ & $1(1.2 \%)$ \\
\hline Lung & $0(0 \%)$ & $5(12.8 \%)$ & $5(6.0 \%)$ \\
\hline Melanoma & $0(0 \%)$ & $2(5.1 \%)$ & $2(2.4 \%)$ \\
\hline Prostate & $0(0 \%)$ & $3(7.7 \%)$ & $3(3.6 \%)$ \\
\hline Renal & $0(0 \%)$ & $1(2.6 \%)$ & $1(1.2 \%)$ \\
\hline Sarcoma & $0(0 \%)$ & $2(5.1 \%)$ & $2(2.4 \%)$ \\
\hline Thymoma & $0(0 \%)$ & $1(2.6 \%)$ & $1(1.2 \%)$ \\
\hline Thyroid & $0(0 \%)$ & $1(2.6 \%)$ & $1(1.2 \%)$ \\
\hline \multicolumn{4}{|l|}{ Cancer Treatment } \\
\hline aCD20 & $9(20.0 \%)$ & $0(0 \%)$ & $9(10.7 \%)$ \\
\hline aCD20 + chemo & $9(20.0 \%)$ & $0(0 \%)$ & $9(10.7 \%)$ \\
\hline anti-CD30 & $1(2.2 \%)$ & $0(0 \%)$ & $1(1.2 \%)$ \\
\hline aHER2 & $0(0 \%)$ & $2(5.1 \%)$ & $2(2.4 \%)$ \\
\hline AXL inhibitor & $1(2.2 \%)$ & $0(0 \%)$ & $1(1.2 \%)$ \\
\hline Bispecific & $1(2.2 \%)$ & $0(0 \%)$ & $1(1.2 \%)$ \\
\hline BTK inhibitor & $4(8.9 \%)$ & $0(0 \%)$ & $4(4.8 \%)$ \\
\hline CAR-T & $1(2.2 \%)$ & $0(0 \%)$ & $1(1.2 \%)$ \\
\hline Chemotherapy & $8(17.8 \%)$ & $15(38.5 \%)$ & $23(27.4 \%)$ \\
\hline EZH inhibitor & $1(2.2 \%)$ & $0(0 \%)$ & $1(1.2 \%)$ \\
\hline PI3K Inhibitor & $1(2.2 \%)$ & $0(0 \%)$ & $1(1.2 \%)$ \\
\hline Proteasome inhibitor & $3(6.7 \%)$ & $0(0 \%)$ & $3(3.6 \%)$ \\
\hline Radiation & $1(2.2 \%)$ & $0(0 \%)$ & $1(1.2 \%)$ \\
\hline Tyrosine kinase inhibitor & $1(2.2 \%)$ & $0(0 \%)$ & $1(1.2 \%)$ \\
\hline Hormonal & $0(0 \%)$ & $5(12.8 \%)$ & $5(6.0 \%)$ \\
\hline Immune checkpoint blockade & $0(0 \%)$ & $7(17.9 \%)$ & $7(8.3 \%)$ \\
\hline VEGF inhibitor & $0(0 \%)$ & $1(2.6 \%)$ & $1(1.2 \%)$ \\
\hline None & $4(8.9 \%)$ & $9(23.1 \%)$ & $13(15.5 \%)$ \\
\hline
\end{tabular}




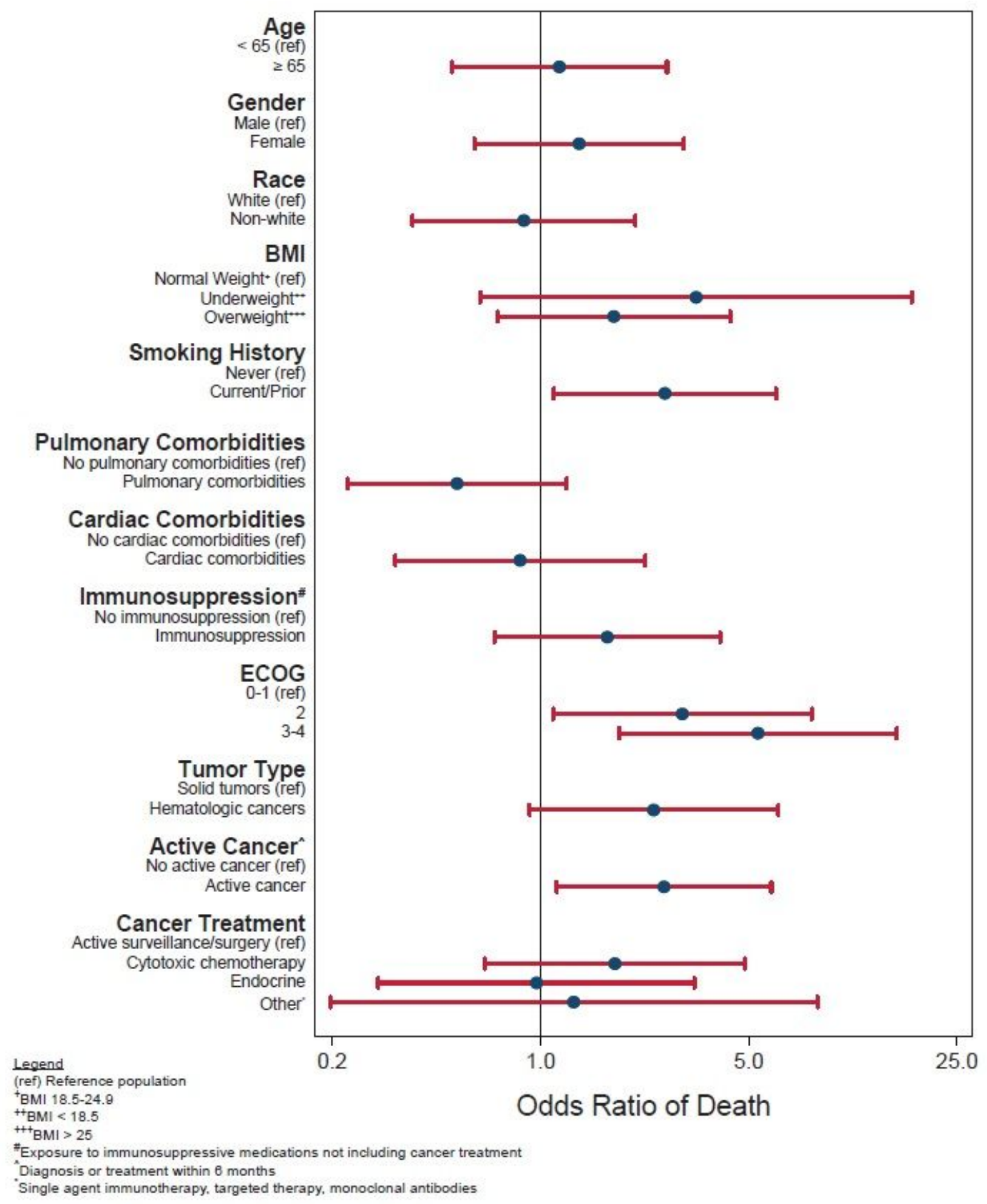

\section{Figure 1}

Univariate analysis of potential risk factors in COVID-19 mortality. 

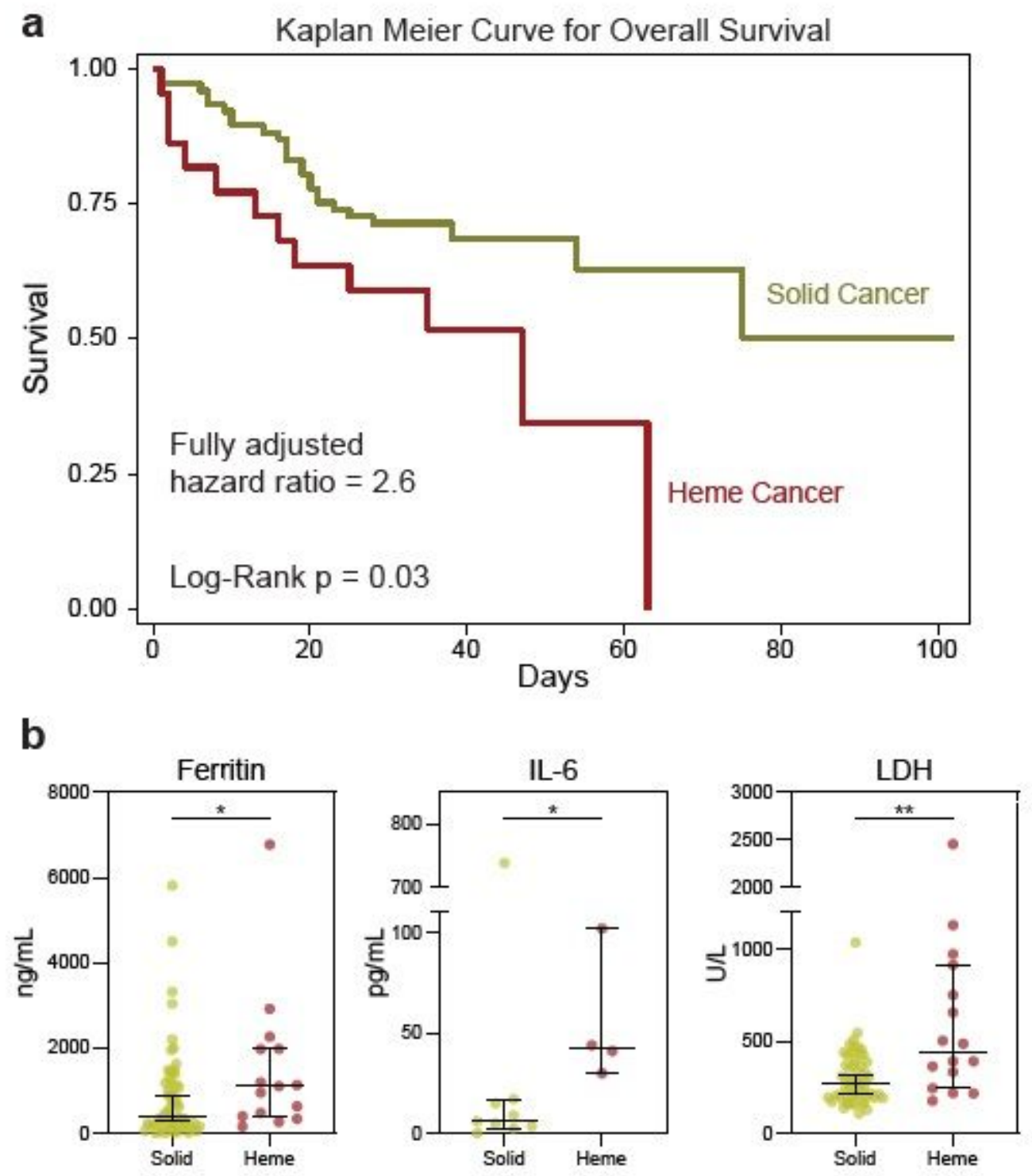

\section{Figure 2}

Hematologic cancer is an independent risk factor for COVID-19 related mortality. (a) Kaplan Meier curve for COVID-19 survival of patients with solid $(n=77)$ and hematologic $(n=22)$ cancer. Cox regressioncomputed hazard ratio for mortality in hematologic vs solid cancer, adjusted for age, gender, smoking status, active cancer status, and ECOG performance status. (b) Ferritin, IL-6, and LDH in solid ( $n=62$ ) and hematologic $(n=15)$ cancer hospitalized for COVID-19. (All) Significance determined by Mann Whitney test: ${ }^{\star} p<0.05,{ }^{\star \star} p<0.01,{ }^{\star \star *} p<0.001$, and ${ }^{\star \star \star \star *} p<0.0001$. Median and $95 \% \mathrm{Cl}$ shown. 
a

\begin{tabular}{|c|c|c|}
\cline { 2 - 3 } & \multicolumn{2}{|c|}{ PENN: MESsI } \\
\cline { 2 - 3 } Number & No Cancer & Active Cancer \\
\cline { 2 - 3 } Age (median) & 60.0 & 22 \\
& & 66.0 \\
Gender Female & $49(45.4 \%)$ & $14(63.6 \%)$ \\
Male & $59(54.6 \%)$ & $8(36.4 \%)$ \\
Black & $74(68.5 \%)$ & $16(72.7 \%)$ \\
Race Caucasian & $28(25.9 \%)$ & $5(22.7 \%)$ \\
Asian & $5(4.6 \%)$ & $1(4.5 \%)$ \\
Mortality (28 days) & $12(11.1 \%)$ & $8(36.4 \%)$ \\
\cline { 2 - 3 }
\end{tabular}

e

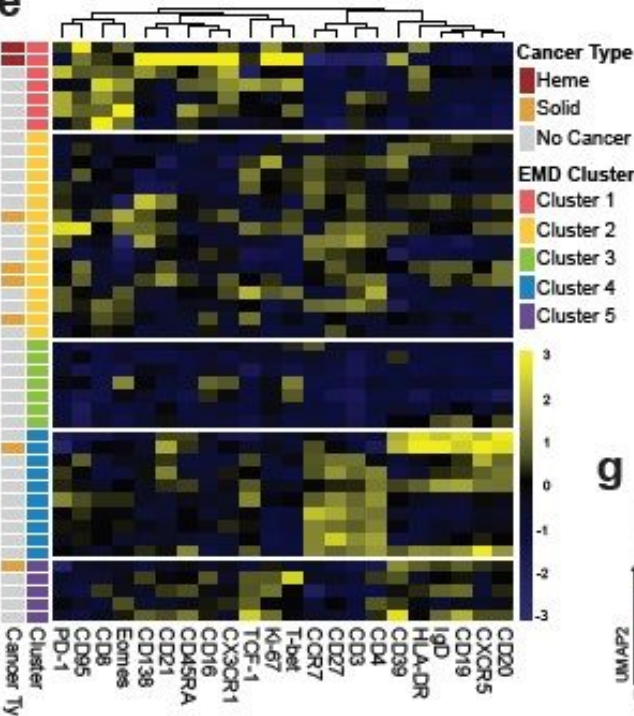

궁

i

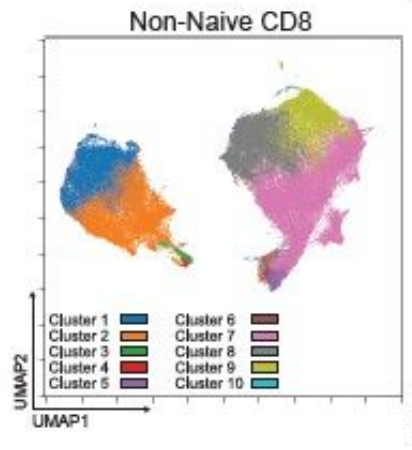

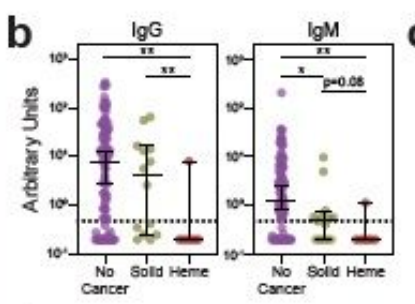
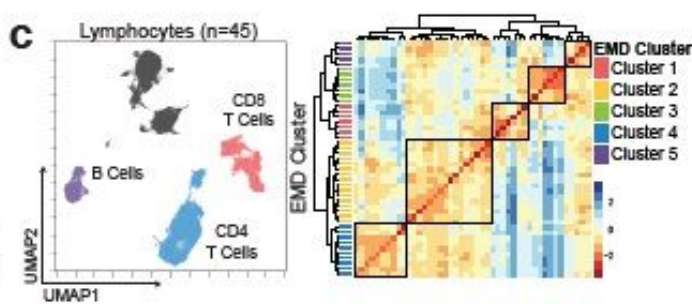

d
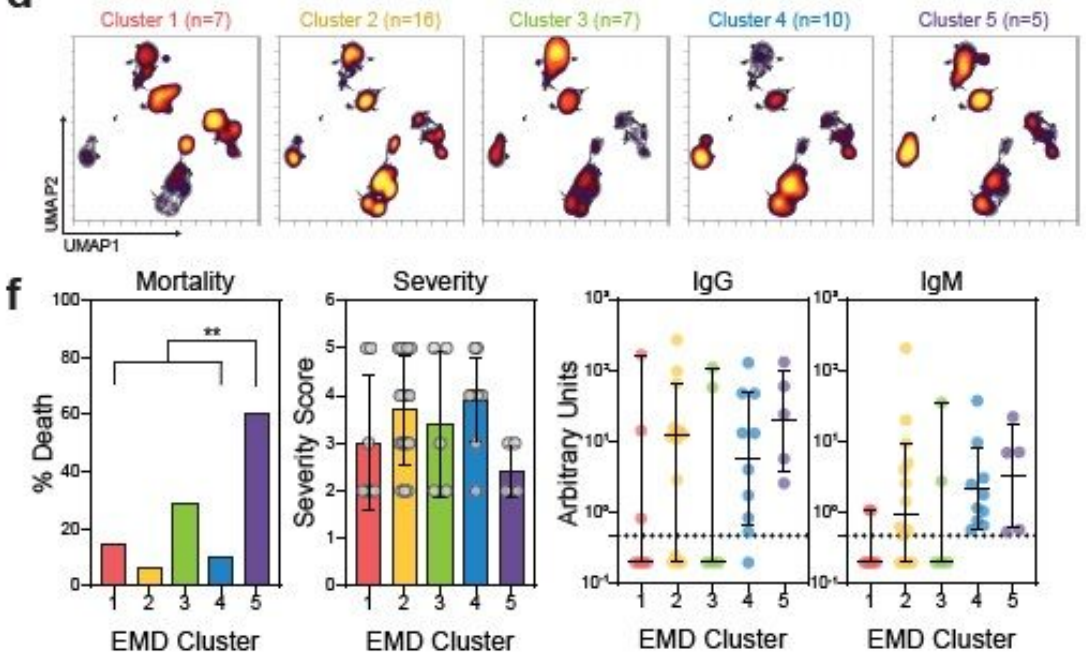

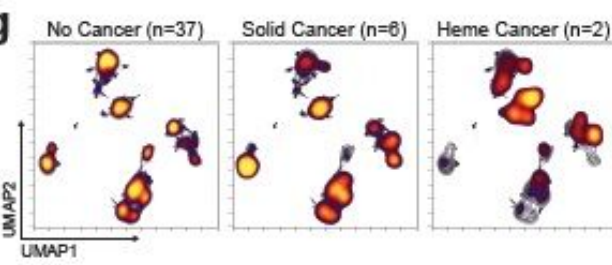

k

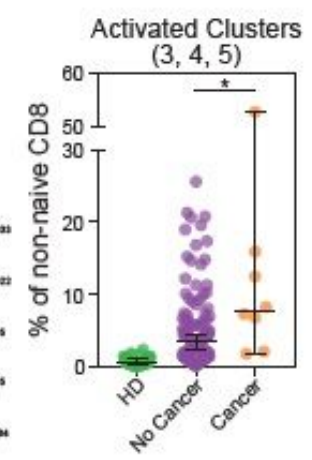

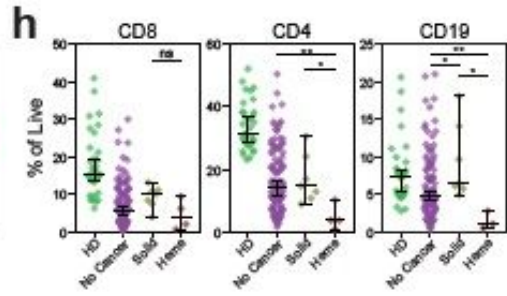

I
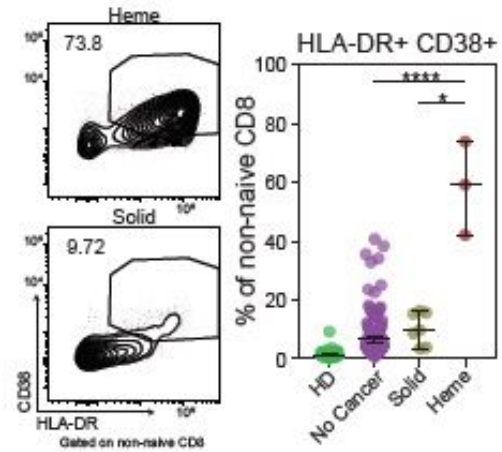

\section{Figure 3}

High dimensional analyses reveal immune phenotypes associated with mortality and distinct phenotypes between solid and hematologic cancers. (a) Demographic and mortality data for MESSI cohort at Penn. (b) Relative levels of SARS-CoV-2 IgG and IgM of solid $(n=14)$ and hematologic $(n=7)$ cancer patients and non-cancer patients $(n=108)$. (c) (Left) Global UMAP projection of lymphocyte populations for all 45 patients pooled. (Right) Hierarchical clustering of Earth Mover's Distance (EMD) using Pearson correlation, calculated pairwise for lymphocyte populations. (d) UMAP projection of concatenated lymphocyte populations for each EMD cluster. (Yellow: High Density; Black; Low Density) 
(e) Heatmap showing expression patterns of various markers, stratified by EMD cluster. Heat scale calculated as column z-score of MFI. (f) Mortality, disease severity, and SARS-CoV-2 antibody data, stratified by EMD cluster (Cluster $5 n=5$; Cluster 1,2,3,4 $n=40$ ). Mortality significance determined by Pearson Chi Square test. Severity assessed with NIH ordinal scale for COVID-19 clinical severity (1: Death; 8: Normal Activity)15. (g) UMAP projections of concatenated lymphocyte populations for solid cancer, hematologic cancer, and non-cancer patients. (h) CD8 and CD4 T cell and B cell frequencies in healthy donors (HD) $(n=33)$, non-cancer $(n=108)$, solid cancer $(n=7)$, and heme cancer $(n=4)$. (i) UMAP projection of non-naive CD8 T cell clusters identified by FlowSOM. (j) (Top) UMAP projections of nonnaïve CD8 T cells for non-cancer and cancer patients. (Bottom) UMAP projections indicating HLA-DR and CD38 protein expression on non-naive CD8 T cells for all patients pooled. (k) Frequency of activated FlowSOM clusters in HD ( $n=30)$, non-cancer $(n=110)$, and cancer patients $(n=8)$. (l) Representative flow plots and frequency of HLA-DR and CD38 co-expression in HD $(n=30)$, non-cancer $(n=110)$, solid cancer $(n=7)$, and hematologic cancer $(n=3)$ patients. (All) Significance determined by Mann Whitney test: ${ }^{*} p<0.05,{ }^{* *} p<0.01, * * * p<0.001$, and ${ }^{* * \star *} p<0.0001$. Median and $95 \% \mathrm{Cl}$ shown. 

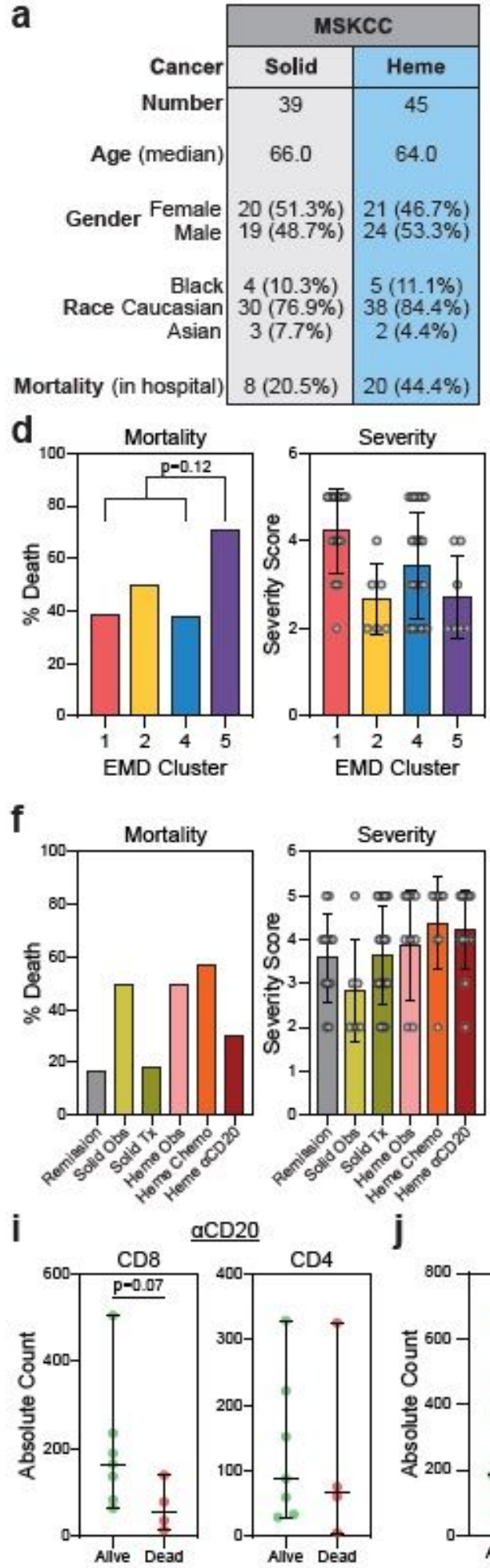

b
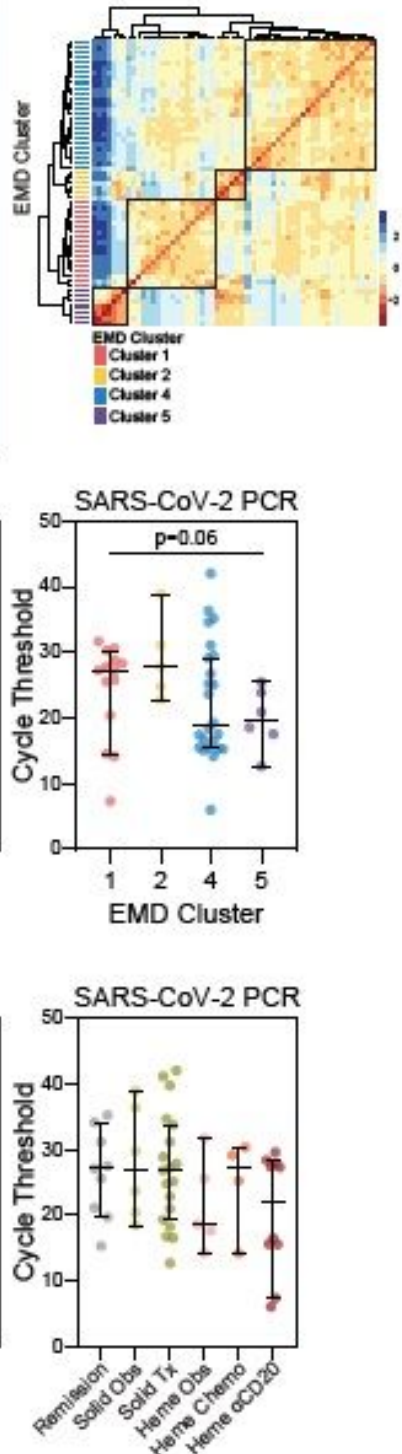

C
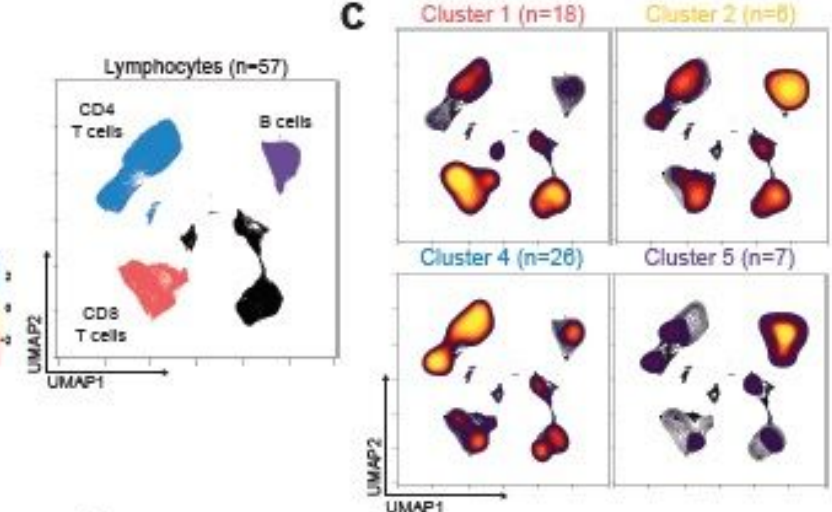

e
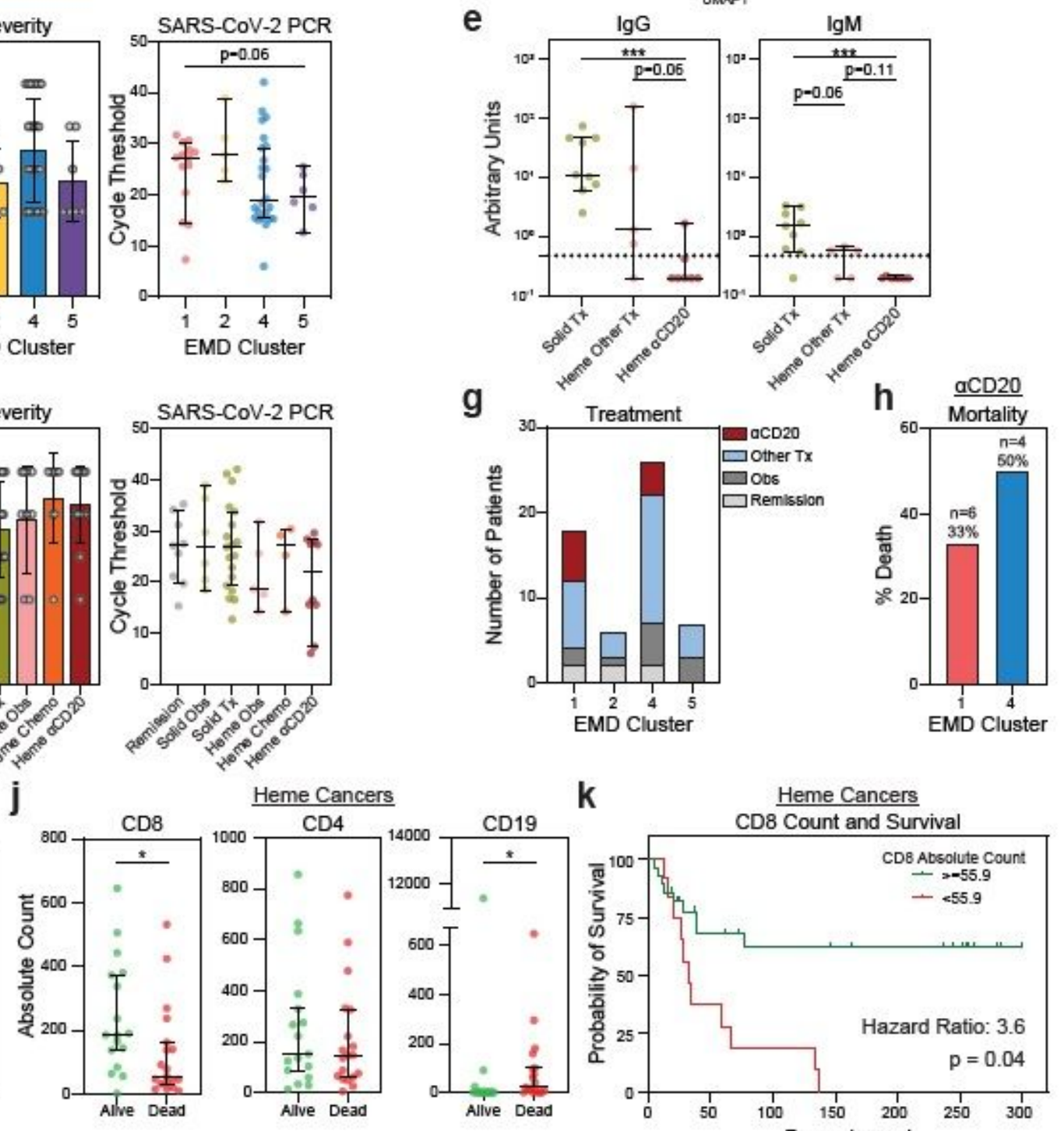

Heme Cancers CD8 Count and Survival

$\mathbf{k}$

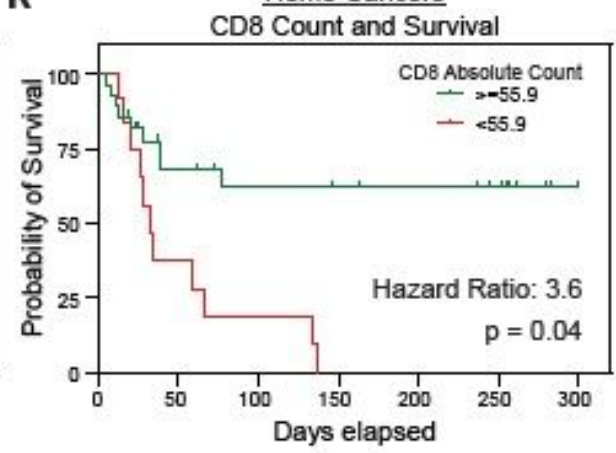

Figure 4

CD8 T cell counts associated with survival in hematologic cancer patients with COVID-19. (a) Demographic and mortality data of MSKCC cohort. (b) (Left) Hierarchical clustering of Earth Mover's Distance (EMD) using Pearson correlation, calculated pairwise for lymphocyte populations. (Right) Global UMAP projection of lymphocyte populations pooled. (c) UMAP projection of concatenated lymphocyte populations for each EMD cluster. (Yellow: High Density; Black: Low Density) (d) Mortality 
(Cluster $5 \mathrm{n}=7$; Cluster 1,2,4 $\mathrm{n}=50$ ), severity, and RT-PCR cycle threshold (Cluster $1 \mathrm{n}=14$; Cluster $2 \mathrm{n}=5$; Cluster $4 \mathrm{n}=24$; Cluster $5 \mathrm{n}=6$ ) (Lower Ct: Higher viral load) stratified by EMD cluster. Mortality significance determined by Pearson Chi Square test. (e) Relative levels of SARS-CoV-2 IgG and IgM of patients with recent cancer treatments (solid tx $n=9$; heme $\operatorname{aCD} 20 n=7$; heme other $\operatorname{tx} n=5$ ). (f) Mortality, severity, and RT-PCR cycle threshold stratified by cancer treatment (remission $n=9$; solid obs $n=6$; solid tx $n=19$; heme obs $n=5$; heme chemo $n=4$; heme $\operatorname{aCD} 20 n=10$ ). Severity assessed with NIH ordinal scale for COVID-19 clinical severity. (g) Recent cancer treatment of patients in each EMD cluster. (h) Mortality of patients treated with B cell depleting therapy in EMD cluster 1 (red) and EMD cluster 4 (blue). (i) Absolute CD8 and CD 4 T cell counts in patients treated with $B$ cell depleting therapy (alive $n=7 ;$ dead $n=4$ ). ( $j$ ) Absolute CD8 and CD4 T cell counts and B cell counts in hematologic cancer patients (alive $n=17$; dead $\mathrm{n}=18$ ). ( $\mathrm{k}$ ) Kaplan-Meier curve for survival in hematologic cancer patients stratified by CD8 $\mathrm{T}$ cell counts (threshold = 55.9; log-rank hazard ratio) ( $>=55.9 n=28 ;<55.9 n=13)$. CD8 count threshold determined by Classification and Regression Tree (CART) analysis. (All) Significance determined by Mann Whitney test: ${ }^{*} p<0.05,{ }^{* *} p<0.01,{ }^{* \star *} p<0.001$, and ${ }^{* \star * *} p<0.0001$. Median and $95 \% \mathrm{Cl}$ shown. 